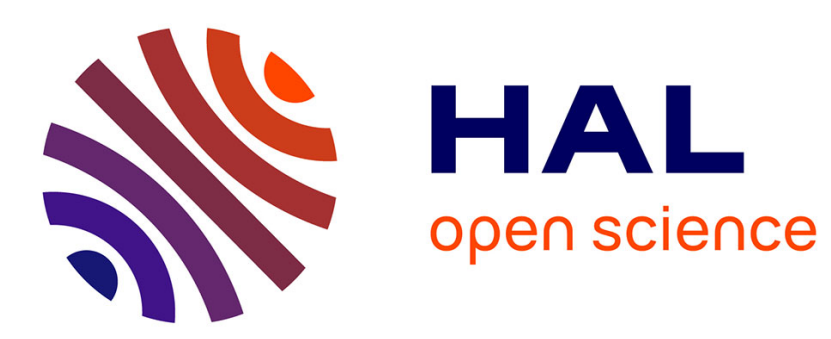

\title{
Reversible Bifurcation of Homoclinic Solutions in Presence of an Essential Spectrum
}

\author{
Matthieu Barrandon
}

\section{To cite this version:}

Matthieu Barrandon. Reversible Bifurcation of Homoclinic Solutions in Presence of an Essential Spectrum. Journal of Mathematical Fluid Dynamics, 2006, Volume 8, Number 2 / April, 2006, pp.267-310. 10.1007/s00021-004-0145-3 . hal-00124989v2

\section{HAL Id: hal-00124989 \\ https://hal.science/hal-00124989v2}

Submitted on 18 Jan 2007

HAL is a multi-disciplinary open access archive for the deposit and dissemination of scientific research documents, whether they are published or not. The documents may come from teaching and research institutions in France or abroad, or from public or private research centers.
L'archive ouverte pluridisciplinaire HAL, est destinée au dépôt et à la diffusion de documents scientifiques de niveau recherche, publiés ou non, émanant des établissements d'enseignement et de recherche français ou étrangers, des laboratoires publics ou privés. 


\title{
Reversible bifurcation of homoclinic solutions in presence of an essential spectrum
}

\author{
Matthieu Barrandon \\ INLN, UMR CNRS-UNSA 6618 \\ 1361 routes des Lucioles, 06560 Valbonne \\ Matthieu.Barrandon@inln.cnrs.fr
}

\begin{abstract}
We consider bifurcations of a class of infinite dimensional reversible dynamical systems which possess a family of symmetric equilibria near the origin. We also assume that the linearized operator at the origin $L_{\varepsilon}$ has an essential spectrum filling the entire real line, in addition to the simple eigenvalue at 0. Moreover, for parameter values $\varepsilon<0$ there is a pair of imaginary eigenvalues which meet in 0 for $\varepsilon=0$, and which disappear for $\varepsilon>0$. The above situation occurs for example when one looks for travelling waves in a system of superposed perfect fluid layers, one being infinitely deep. We give quite general assumptions which apply in such physical examples, under which one obtains a family of bifurcating solutions homoclinic to every equilibrium near the origin. These homoclinics are symmetric and decay algebraically at infinity, being approximated at main order by the Benjamin - Ono homoclinic. For the water wave example, this corresponds to a family of solitary waves, such that at infinity the upper layer slides with a uniform velocity, over the bottom layer (at rest).
\end{abstract}

Key words: bifurcation theory, nonlinear water waves, homoclinic orbits, solitary waves, infinite-dimensional reversible dynamical systems, essential spectrum.

AMS classification: 34C23, 35A20, 35B32, 35J60, 76B15, 76B25

\section{Introduction}

For solving elliptic problems in strips, the use of a "spatial dynamics" formulation was introduced in the 80's by K. Kirchgässner [9]. Choosing the spatial coordinate $x$ as a "time" coordinate, the initial value problem is then ill-posed, but since we are looking for solutions bounded on all the real line, this leads to a sort of "boundary value" problem. When the problem has a reflection symmetry $x \rightarrow-x$, this leads to a reversible dynamical system for which the vector field anticommutes with a symmetry $S$. An easy consequence is that if $U(x)$ is a solution then $S U(-x)$ is also a solution. Very fruitful applications in infinite dimensional systems were found in hydrodynamical problems, where the reflection symmetry results from the geometry of 
the flow. For the search of non trivial solutions of Navier Stokes equations in a cylindrical domain (see for instance [8]), heteroclinic orbits correspond to defect solutions connecting two symmetric equilibria. For solutions of various water wave problems (see for instance the review paper [4]), the search of travelling waves corresponds to solutions which are steady in a horizontally moving reference frame and a homoclinic orbit corresponds to a solitary wave.

In the present work we consider bifurcations of a class of infinite dimensional reversible dynamical systems, having general features occurring in water wave problems, when one looks for travelling waves, in a set of superposed layers, the bottom one being infinitely deep. Our system has the following form

$$
\frac{d U}{d x}=L_{\varepsilon} U+N_{\varepsilon}(U),
$$

where $U(x)$ lies in a function space, $U=0$ is an equilibrium solution for all parameter values $\varepsilon \in \mathbb{R}, L_{\varepsilon}$ is a linear operator depending on $\varepsilon$, and $N_{\varepsilon}$ is a nonlinear operator, at least quadratic in $U$. We assume that the linearized operator at the origin $L_{\varepsilon}$ has an essential spectrum filling the entire real line. We show in section 2 how to derive (1.1) for a typical example taken in water wave theory. Our problem concerns more precisely reversible dynamical systems as (1.1) such that in addition to the essential spectrum filling the entire real line, the operator $L_{\varepsilon}$ has a simple eigenvalue at 0 . The occurrence of such an eigenvalue corresponds to the existence of a one parameter family of reversible equilibria for (1.1). Moreover, the bifurcation phenomenon comes from the assumption that for parameter values $\varepsilon<0$ there is a pair of imaginary eigenvalues which meet in 0 for $\varepsilon=0$ and which disappear for $\varepsilon>0$ (see Figure 1).
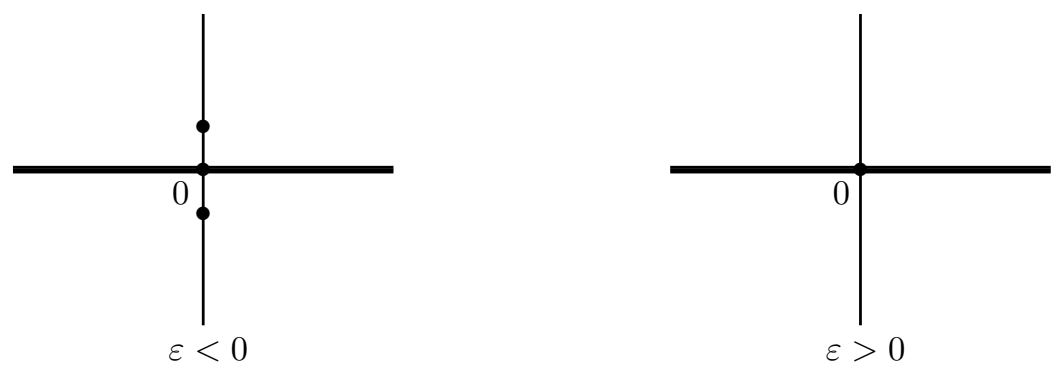

Figure 1: Spectrum of $L_{\varepsilon}$ (before rescaling)

In the general theory of bifurcations of reversible dynamical systems classical results are known in the cases when the system can be reduced to a finite dimensional ordinary differential equation by using a center manifold reduction method. This leads to the study of a perturbed normal form (see for instance the review paper [4]). For applying this method, the linear operator $L_{\varepsilon}$ must have a spectral gap near the imaginary axis. In such a case the description of the finite spectrum near the imaginary axis is sufficient to understand the dynamics of small reversible solutions of (1.1).

Since the entire real axis belongs to the spectrum, there is no spectral gap in our problem and we cannot use a center manifold reduction theorem. The absence 
of spectral gap is not a big problem when one looks for periodic solutions, since quite simple conditions on the nonlinear term still lead to the same result as in finite dimensions (Lyapunov - Devaney theorem) (see [5] for typical results for travelling water waves).

The same type of spectrum as in our present case, appears in the works [6] and [7], where two different water wave problems are treated, looking for solitary waves or generalized solitary waves.

In [6] the authors consider one infinitely deep layer of perfect fluid, with surface tension on the free surface. In this work, the bifurcation comes from a pair of double imaginary eigenvalues splitting into two pairs of eigenvalues (then not meeting the essential spectrum on the real axis). The principal part of the bifurcating homoclinic solutions, is given at finite distance by homoclinic solutions of a four dimensional reversible ordinary differential equation, while the influence of the essential spectrum is only felt when $|x| \rightarrow \infty$ by a polynomial decay (instead of exponential decay).

In [7] the authors consider two superposed layers, the bottom one being deep, and with no surface tension at the free surface and at the interface. The bifurcation comes, as in the present work, from the merging of two imaginary eigenvalues $(\varepsilon<0$ tending towards 0 ) which disappear in the point 0 of the essential spectrum when $\varepsilon$ becomes positive. In [7] there is a additional pair of simple imaginary eigenvalues at a distance $O(1)$ from 0 for all values of the parameter $\varepsilon$ leading to more complications than in the present work. In the case of [7], the existence of bifurcating generalized solitary waves, with polynomial decay at infinity, is proved. In fact, there is a family of periodic solutions and a family of homoclinic connections to these periodic orbits provided that they are not too small (as it is expected when a slow decaying dynamics competes with a fast oscillation). In the present work, we have no additional imaginary eigenvalues, hence we do not expect the occurrence of the above phenomenon. Since the work [7] is very technical, and specific to the above physical situation, our aim is to consider a simpler situation in a more general frame. We provide two examples of application belonging to the theory of water-waves.

The first example is when one considers two layers of superposed perfect fluids with no surface tension at the interface, assuming that the flow is potential and subject to gravity. We assume that the upper layer is bounded by a rigid horizontal top and the bottom one is infinitely deep. We are interested in travelling waves of horizontal velocity $c$. The existence of solitary waves with polynomial decay at infinity has been obtained independently by Amick [1] and Sun [12]. The solitary wave (see Figure 1), corresponding to a homoclinic solution in a dynamical system approach, is approximated at first order by the Benjamin-Ono solitary wave (see (1.4) below). We prove here that this result can be derived as well by our method. In addition, we complete their result by the existence of a one parameter family of solitary waves, for which the flow at infinity corresponds to a uniform sliding of the upper layer over the bottom one (the additional parameter is the uniform velocity of the upper layer at infinity).

A second example of application is when one considers two layers of perfect fluid with the same geometry as before but with a free upper surface with sufficiently large surface tension, and with no surface tension at the interface. With the theory presented here we show the existence of a one parameter family of solitary waves for this problem (see Figure 1) which corresponds to a family of homoclinic solutions 


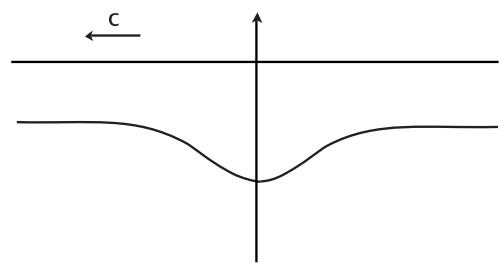

Figure 2: Shape of the solitary wave for example 1

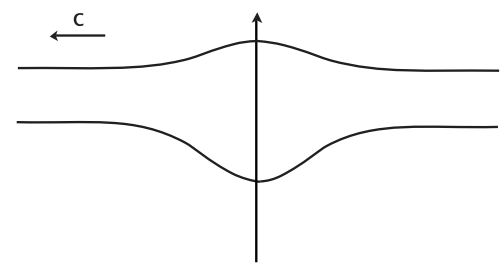

Figure 3: Shape of the solitary wave for example 2

for the reversible dynamical system. Physically, here again the additional parameter corresponds to a uniform flow at infinity of the upper layer over the bottom one, at rest.

We believe that other examples in water wave theory with an infinitely deep stratified layer of an incompressible fluid with or without a free surface, might enter into the frame developed below.

Instead of concentrating on a new example, we prefer to give quite general assumptions, which apply in particular on the two examples mentioned above, and which lead to bifurcated solutions like a family of homoclinics with polynomial decay at infinity.

Other problems dealing with bifurcations in presence of a continuous spectrum can be found for instance in the work of Stuart [11] where bifurcations occur out to the end of a continuous spectrum. This type of situation happens here if one considers the linear operator $Q_{\varepsilon}: \frac{d U}{d x}-L_{\varepsilon} U$ in a space of functions $x \mapsto U(x)$ tending towards 0 at infinity. In our problem $Q_{\varepsilon}$ is invertible for $\varepsilon>0$ (see lemma 4.4 of section 4.3) while it is not invertible for $\varepsilon \leq 0$. This property might appear as a sort of "continuous spectrum" in $\varepsilon \leq 0$. A major difference with Stuart's work is that our $Q_{\varepsilon}$ is not self-adjoint, and that we make instead assumptions mainly on the resolvent of $L_{\varepsilon}$ near 0 on the imaginary axis.

The sole description of the spectrum is not sufficient anymore for understanding the singularity at $k=0$ of the resolvent operator $\left(i k-L_{\varepsilon}\right)^{-1}$. Therefore we must make suitable assumptions on this operator (for $k$ real), to characterize the singularities introduced by the essential spectrum occurring on the real axis near 0 .

First we need to make a rescaling in coordinates and variables which dilates the original spectrum by a factor $1 / \varepsilon$ and gives the new linear operator $\mathcal{L}_{\varepsilon}$, the new nonlinear term is denoted by $\mathcal{N}_{\varepsilon}$. The best way is then to describe the resolvent operator $\left(i k-\mathcal{L}_{\varepsilon}\right)^{-1}$. It is known that a simple isolated eigenvalue of $\mathcal{L}_{\varepsilon}$ corresponds to a simple pole of the resolvent. When the essential spectrum of $\mathcal{L}_{\varepsilon}$ contains 0 , the singularity is more complicated and needs to be specified. This is the purpose of section 3 and in particular Hypothesis H1 (see section 3.2.1). The main assumption in $\mathrm{H} 1$ is that we can write the resolvent for $k$ real, $|k|$ small $\neq 0$, as

$$
\left(i k-\mathcal{L}_{\varepsilon}\right)^{-1} V=\frac{\xi_{\varepsilon}^{*}(V)}{i k \varepsilon \Delta} \xi_{0}+\frac{\eta_{\varepsilon}^{*}(V)}{\Delta} \theta_{k}+\ldots
$$

Here $\xi_{0}$ is the eigenvector associated with the 0 eigenvalue and $i k \varepsilon \Delta(k, \varepsilon)=0$ is the usual "dispersion equation" in physical problems like in water wave theory, the linear 
forms $\xi_{\varepsilon}^{*}$ and $\eta_{\varepsilon}^{*}$ defining the closure of the range of $\mathcal{L}_{\varepsilon}$ (classically $V \mapsto \frac{\xi_{\varepsilon}^{*}(V)}{\varepsilon \Delta(0, \varepsilon)}$ is the projection on the eigenvector $\xi_{0}$, commuting with $\mathcal{L}_{\varepsilon}$ ). We also assume that (for $k$ real)

$$
\Delta(\varepsilon, k)=1+a|k|+O\left(\varepsilon k^{2}\right),
$$

where $a>0$. Note that before the scaling, the dispersion relation takes the form $\varepsilon+a|k|+$ h.o.t. for $\varepsilon$ and $k$ close to 0 , leading to a pair of imaginary eigenvalues $i k \sim \pm i \varepsilon / a$ for $\varepsilon<0$. So, the present form of $\Delta$ keeps the track of this property, even though there is no longer any imaginary eigenvalue for $\varepsilon>0$. We describe in $\mathrm{H} 1$ and $\mathrm{H} 2$ the singularities introduced by the essential spectrum. These singularities appear in $\Delta$, in the vector $\theta_{k}$ and also in the linear forms introduced when we write the resolvent according to the previous formula. Hence we need to introduce some function spaces to describe the behavior of the resolvent in 0 . The vector $\theta_{k}$ plays an important role in the construction of the homoclinic solutions. Indeed we prove that this construction essentially depends on the operator

$$
u \mapsto \mathcal{T}(u)=\mathcal{F}^{-1}\left(-i k \hat{u} \theta_{k}\right),
$$

where $\hat{u}$ denotes the Fourier transform $\mathcal{F}(u)$ of a function $u$. We also make an assumption on the "regularity" of this operator (Hypothesis H2) after having introduced some spaces which describe this regularity. In particular, we suppose that for a smooth enough function $u$ decaying as $1 /|x|^{2}$ at infinity, then $\mathcal{T}(u)$ lies in a very specific space. In such a space, for the examples mentioned above, the decay rates in the $x$ variable and in the vertical variable $y$ are linked, and for a fixed value of $y, U(x)$ decays as $1 /|x|^{2}$ at infinity. We finally suppose that the resolvent behaves like $1 / k$ when $k$ is real, $|k|$ large (Hypothesis $\mathrm{H} 3$ ).

In section 4 we study the bifurcation problem mentioned before. We first describe in $\mathrm{H} 4$ the required regularity of the nonlinear term in the spaces introduced in section 3 and give with $\mathrm{H} 5$ a more specific assumption

$$
\xi_{\varepsilon}^{*}\left(D_{U U} \mathcal{N}_{\varepsilon \mid U=0}\left(\xi_{0}, \theta_{k}\right)=c_{0}+O(\varepsilon), \quad c_{0} \neq 0,\right.
$$

satisfied in our examples. We then look for homoclinic solutions to 0 with polynomial decay as $|x| \rightarrow \infty$. This slow decay leads to many complications which would not appear with exponential decaying functions. This is the purpose of section 4 to prove the existence of homoclinic connections to 0 with an algebraic decay at infinity, which are approximated by a Benjamin-Ono solitary wave.

Result of Theorem 4.3. Under general hypothesis on $\mathcal{L}_{\varepsilon}$ (see H1, H2 and H3) and on $\mathcal{N}_{\varepsilon}$ (see $H_{4}$ and H5) made precise below, there exists $\varepsilon_{0}>0$ and $\nu_{0}>0$, such that, for any $0<\varepsilon<\varepsilon_{0}, 0 \leq|\nu|<\nu_{0} \varepsilon$, equation (1.1) has a weak reversible homoclinic connection $U_{\varepsilon, \nu}$ to 0 which satisfies

$$
U_{\varepsilon, \nu}(x)=\varepsilon\left(\nu+u_{h}(\varepsilon x)\right) \xi_{0}+\widetilde{U}(\varepsilon, \nu),
$$

where $\varepsilon \nu \xi_{0}$ is the family of equilibria, solutions of (1.1), $u_{h}$ is the Benjamin-Ono homoclinic connection $(O(1))$ (see equation (1.4)) given by

$$
u_{h}(x)=\frac{-2}{a c_{0}\left(1+(x / a)^{2}\right)},
$$


and $\xi_{0}$ is the (symmetric) eigenvector associated with the 0 eigenvalue. The term $\widetilde{U}(\varepsilon, \nu)=O\left(\varepsilon^{2}+|\nu|\right)$ decays polynomialy at infinity. For the water wave examples, the form (1.2) is still valid for $0 \leq|\nu|<\nu_{0}$, and $\widetilde{U}(\varepsilon, \nu)=O\left(\varepsilon^{2}+\varepsilon|\nu|\right)$.

To prove this result we use a reduction strategy. Indeed we look for solutions written as

$$
U=\varepsilon(\nu+w) \xi_{0}+\varepsilon^{2} Y,
$$

where $\nu$ is a scalar, $w$ a scalar function and $Y$ lies in a subspace complementary to $\xi_{0}$, and we reduce our problem to an equation for the scalar function $w$, in proving that $Y$ can be written as a function of $w$ (see Theorem 4.9 in section 4.3.2):

Reduction Theorem. Under the hypotheses H1, H2 and H3 on $\mathcal{L}_{\varepsilon}$ and hypothesis $H_{4}$ on $\mathcal{N}_{\varepsilon}$ made precise below, there exist $\varepsilon_{0}$ and $\nu_{0}>0$ such that, for $0<\varepsilon<\varepsilon_{0}$, $|\nu|<\nu_{0}|\ln \varepsilon|^{-1}$ and if $U=\varepsilon(\nu+w) \xi_{0}+\varepsilon^{2} Y$ gives a reversible solution of (1.1) tending towards 0 at infinity, then

$$
Y=Y_{\varepsilon, \nu}(w)=a \mathcal{T}(w)+O(\varepsilon+|\nu||\ln \varepsilon|) .
$$

This result corresponds to the reduction of (1.1) to a scalar equation for $\nu, Y$ being determined by a function of $w$. It can be seen as a sort of non local center manifold reduction. However, $Y$ lies in a space weaker than required for verifying (1.1) in the basic space. Indeed $N_{\varepsilon}(U)$ takes its values in the basic space, but $L_{\varepsilon} U$ lies in a larger space, and is defined via its Fourier transform. In our examples, this weak sense automatically leads to a system satisfied in the strong sense, thanks to the elliptic properties of the Cauchy-Riemann system in the half space.

Note that hypothesis H5 (see below) is not required in this reduction theorem. We then have to find the equation satisfied by $w$ (see Lemma 4.10 in section 4.3.3):

Equation for $\boldsymbol{w}$. Assume that $\mathcal{L}_{\varepsilon}$ satisfies hypotheses H1, H2 and H3 and that $\mathcal{N}_{\varepsilon}$ satisfies hypotheses $H_{4}$ and $H 5$. Let $U=\varepsilon(\nu+w) \xi_{0}+\varepsilon^{2} Y$ be a reversible solution of (1.1) tending towards 0 at infinity. Then $w$ satisfies

$$
w+a \mathcal{H}\left(w^{\prime}\right)+a c_{0} w^{2}=O(\varepsilon+|\nu| / \varepsilon),
$$

where $\mathcal{H}$ is the Hilbert transform (see (3.2)).

Hypothesis $\mathrm{H} 5$ is used in this result to give a local non zero non linear term $\left(a c_{0} w^{2}\right)$ in the bifurcation equation. This scalar equation is a perturbation of the (non-local) Benjamin-Ono equation

$$
w+a \mathcal{H}\left(w^{\prime}\right)+a c_{0} w^{2}=0,
$$

introduced by Benjamin ([3]) and Ono ([10]). We know that this equation admits a solution homoclinic to 0 , given explicitly by (1.3). We then use a perturbation technique for obtaining the result of theorem 4.3.

\section{Examples}

In this section we give examples of the situation described in the introduction. These examples are taken from the theory of water-waves. In particular we show how we can obtain a "spatial dynamics" formulation (see [9]) to describe these problems. 


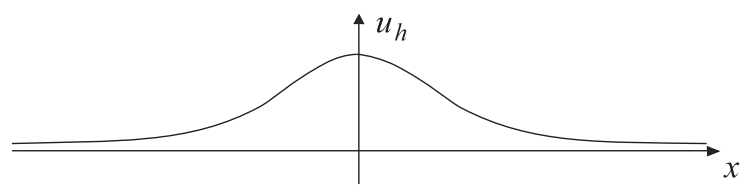

Figure 4: Benjamin-Ono homoclinic for $a c_{0}<0$

The first example consists in two layers of perfect fluids assuming that there is no surface tension at the interface and assuming that the flow is potential and subject to gravity. We also assume that the upper layer is bounded by a rigid horizontal top. This case was treated, using different formulations by Amick [1] and Sun [12]. Since the spatial dynamics formulation we use is very similar to the one used in the second example, we only present the details for the second example.

The second example concerns two layers of perfect fluids (densities $\rho_{1}$ (upper layer) and $\rho_{2}$ (bottom layer)) assuming that there is no surface tension at the interface and assuming that the flow is potential and subject to gravity. Contrary to the first example, we now assume that there is a free upper surface with surface tension $T$. The thickness at rest of the upper layer is $h$ while the bottom one has infinite thickness. We are interested in travelling waves of horizontal velocity $c$. The dimensionless parameters are $\rho=\rho_{1} / \rho_{2} \in(0,1)$ and

$$
\left.\left.\lambda=\frac{g h}{c^{2}} \text { (inverse of (Froude number) }\right)^{2}\right), \quad b=\frac{T}{\rho_{1} h c^{2}} \text { (Weber number). }
$$

In the moving reference frame, denoting by $\xi, \eta$ the physical coordinates, the complex potential in layer $j$ is denoted $w_{j}(\xi+i \eta)$ and the complex velocity $w_{j}^{\prime}(\xi+i \eta)=u_{j}-i v_{j}$. For formulating the problem as a dynamical system we proceed as in [5] and [7] and use the change of coordinates used by Levi-Civita : the new unknown are $\alpha_{j}+i \beta_{j}$, $j=1,2$ which are analytic functions of $w_{j}=x_{j}+i y$ where $x_{j}$ is the velocity potential in the layer $j$ and $y$ is the stream function and where

$$
w_{j}^{\prime}(\xi+i \eta)=e^{\beta_{j}-i \alpha_{j}} .
$$

Notice that $\alpha_{j}$ is the slope of the streamline and $e^{\beta_{j}}$ is the modulus of the velocity in the region $j$. The interface is then given by $y=0$ and the free surface by $y=1$. The region of the flow is $-\infty<y<0$ for fluid 2 and $0<y<1$ for fluid 1 . We choose as the basic $\underline{x}$ coordinate the one given by the bottom layer $\left(x_{2}\right)$ (and we notice that $d x_{1} / d x_{2}=e^{\beta_{10}-\beta_{20}}$ which introduces a factor in the Cauchy-Riemann equations of the upper layer).

With this choice of coordinates we formulate this problem as a dynamical system (see [5])

$$
\frac{d \underline{U}}{d \underline{x}}=L_{\varepsilon} \underline{U}+N_{\varepsilon}(\underline{U}) .
$$

with the following unknown

$$
[\underline{U}(x)](y)=\left(\beta_{20}(\underline{x}), Z(\underline{x}), \alpha_{11}(\underline{x}), \alpha_{1}(\underline{x}, y), \beta_{1}(\underline{x}, y), \alpha_{2}(\underline{x}, \underline{y}), \beta_{2}(\underline{x}, \underline{y})\right)^{t},
$$


where $1+Z(\underline{x})$ is the free surface, $\varepsilon$ is a distinguished parameter defined below and, for example, $\alpha_{1 j} j=0,1$ means the trace of $\alpha_{1}$ in $y=0,1$, and the same convention holds for $\beta_{i j}$. The right hand side of (2.1) is given by

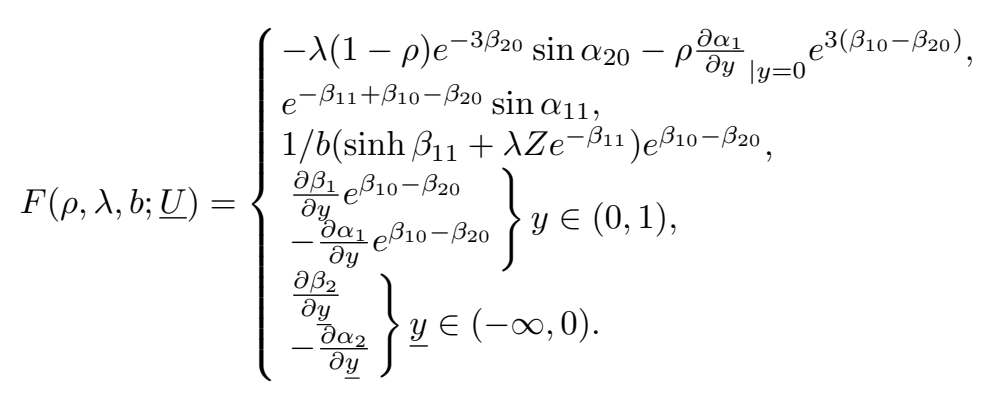

The Galilean invariance of the physical problem induces a reflection symmetry (through the $y$ axis) of the system in the moving frame. This reflection leads to the reversibility of system (2.2), i.e. to the existence of a linear symmetry $S$ which anticommutes with the vector field $F(\lambda, \rho, b ;$.$) . This reversibility symmetry is then defined by$

$$
S \underline{U}=\left(\beta_{20}, Z,-\alpha_{11},-\alpha_{1}, \beta_{1},-\alpha_{2}, \beta_{2}\right)^{t} .
$$

Equation (2.2) is understood in $\mathbb{H}$ where

$$
\mathbb{H}=\mathbb{R}^{3} \times\left\{\mathcal{C}^{0}(0,1)\right\}^{2} \times\left\{\mathcal{C}_{1}^{0}\left(\mathbb{R}^{-}\right)\right\}^{2},
$$

and $\underline{U}(\underline{x})$ lies in $\mathbb{D}$ where

$$
\begin{aligned}
\mathbb{D}= & \mathbb{R}^{3} \times\left\{\mathcal{C}^{1}(0,1)\right\}^{2} \times\left\{\mathcal{C}_{1}^{1}\left(\mathbb{R}^{-}\right)\right\}^{2} \\
& \cap\left\{\alpha_{1}(0)=\alpha_{2}(0), \alpha_{11}=\alpha_{1}(1), \beta_{20}=\beta_{2}(0)\right\},
\end{aligned}
$$

where we define the following Banach spaces

$$
\begin{aligned}
& \mathcal{C}_{1}^{0}\left(\mathbb{R}^{-}\right)=\left\{f \in \mathcal{C}^{0}\left(\mathbb{R}^{-}\right) ; \sup _{y \in \mathbb{R}^{-}}|f(y)|(1+|y|)<\infty\right\}, \\
& \mathcal{C}_{1}^{1}\left(\mathbb{R}^{-}\right)=\left\{f \in \mathcal{C}_{1}^{0}\left(\mathbb{R}^{-}\right) ; f^{\prime} \in \mathcal{C}_{1}^{0}\left(\mathbb{R}^{-}\right)\right\} .
\end{aligned}
$$

The norm in $\mathbb{H}$ for $V=\left(a_{1}, a_{2}, a_{3}, f_{1}, g_{1}, f_{2}, g_{2}\right)^{t} \in \mathbb{H}$ is defined by

$$
\|V\|_{\mathbb{H}}=\left|a_{1}\right|+\left|a_{2}\right|+\left|a_{3}\right|+\left\|f_{1}\right\|_{\infty}+\left\|g_{1}\right\|_{\infty}+\left\|f_{2}\right\|_{1, \infty}+\left\|g_{2}\right\|_{1, \infty},
$$

with

$$
\|f\|_{1, \infty}=\sup _{y \in \mathbb{R}^{-}}|f(y)|(1+|y|)
$$

and we obtain the norm in $\mathbb{D}$ by adding the norms of $f_{i}^{\prime}$ and $g_{i}^{\prime}$.

The dispersion equation reads (for Re $k>0$ )

$$
\begin{aligned}
\Delta_{1}(k)= & k \cosh (k)\left(\rho b k^{2}+\lambda-k\right) \\
& -\sinh (k)\left\{\left(\lambda+b k^{2}\right)[\lambda(1-\rho)-k]+\rho k^{2}\right\}=0,
\end{aligned}
$$


which should be completed by the symmetric relationship for Re $k<0$. The complex roots of $\Delta_{1}(k)=0$ give all complex eigenvalues $i k$ of the linearized operator $D_{\underline{U}} F(\rho, \lambda, b ; 0)=L_{\varepsilon}$ belonging to the upper part of the complex plane. These isolated eigenvalues have a finite multiplicity, and are completed by the symmetric eigenvalues in the lower half plane. They are located in a sectorial region of the complex plane, centered on the real axis, which leads to the finiteness of the number of such eigenvalues in the neighborhood of the imaginary axis. In addition to this discrete set, the spectrum of the linearized operator contains an essential spectrum formed by the entire real axis. This is shown, for example in [5], and this results from the form of the Cauchy-Riemann operator in the infinite layer $(-\infty<y<0)$. Let us give more precisions on the eigenvalues lying on the imaginary axis.

Here $\varepsilon$ is defined by $\varepsilon=1-\lambda(1-\rho)$. We observe that the equation $\Delta_{1}(k)=0$ for $k$ real is equivalent to the equation $\tanh (k)=f(k)$ where $f$ is the rational fraction

$$
f(k)=\frac{k\left(\rho b k^{2}+\lambda-|k|\right)}{\left(\lambda+b k^{2}\right)[\lambda(1-\rho)-|k|]+\rho k^{2}},
$$

and the study of $f$ for $b$ large leads to the following conclusion: for $\varepsilon>0$ small enough, $b$ large enough and $1-\rho=(\alpha / b)^{1 / 3}$ with $0<\alpha<4$ then 0 is the only real solution of the dispersion equation (2.3). This means that for $\varepsilon<0$ there is a pair of eigenvalues on the imaginary axis in addition to the 0 eigenvalue, and for $\varepsilon>0$ this pair disappears. Notice that the case treated in [7] is such that $b=0$, which implies the occurrence of another pair of simple imaginary eigenvalues (given by $k= \pm \lambda$ ) for all values of $\varepsilon$.

Looking at (2.2), we notice the existence of a one parameter family of solutions

$$
\left(0,1 / 2 \lambda\left(1-e^{2 u}\right), 0,0, u, 0,0\right)^{t}, u \in \mathbb{R},
$$

of the nonlinear system (2.1). This family of equilibria corresponds physically to a sliding with a non zero small and uniform velocity of the upper layer over the bottom one. This leads (in differentiating with respect to $u$ ) to the following eigenvector belonging to a zero eigenvalue of the linearized operator (about 0$) L_{\varepsilon}$

$$
\xi_{0}=\left(0, \lambda^{-1}, 0,0,-1,0,0\right)^{t} .
$$

Actually, with the change of variable (changing $\underline{U}$ in $\underline{\widetilde{U}}$ )

$$
Z=\frac{1}{2 \lambda}\left(1-e^{-2 \lambda \widetilde{Z}}\right),
$$

the family of trivial solutions reads $\underline{\widetilde{U}}=u \xi_{0}$.

Let us introduce the basic rescaling of our system for $\varepsilon>0$, hence hiding the pair of imaginary eigenvalues occurring for $\varepsilon<0$. We set

$$
\begin{aligned}
& \varepsilon \underline{x}=x ; \varepsilon \underline{y}=y, y \in(-\infty, 0) ; y=y, y \in(0,1) \\
& \underline{\widetilde{U}}=\varepsilon U,
\end{aligned}
$$

and equation (2.1) now reads

$$
\frac{d U}{d x}=\mathcal{L}_{\varepsilon} U+\mathcal{N}_{\varepsilon}(U),
$$


where

$$
\begin{gathered}
U=\left(\beta_{20}, \widetilde{Z}, \alpha_{11}, \alpha_{1}, \beta_{1}, \alpha_{2}, \beta_{2}\right)^{t}, \\
\mathcal{L}_{\varepsilon} U=\left(\begin{array}{l}
\varepsilon^{-1}\left\{-(1-\varepsilon) \alpha_{10}-\rho{\frac{\alpha \alpha_{1}}{\partial y}}_{\mid y=0}\right\} \\
\varepsilon^{-1} \alpha_{11} \\
\varepsilon^{-1} 1 / b\left(\beta_{11}+\lambda \widetilde{Z}\right) \\
\varepsilon^{-1} \frac{\partial \beta_{1}}{\partial y} \\
-\varepsilon^{-1} \frac{\partial \alpha_{1}}{\partial y} \\
\frac{\partial \beta_{2}}{\partial y} \\
-\frac{\partial \alpha_{2}}{\partial y}
\end{array}\right),
\end{gathered}
$$

and

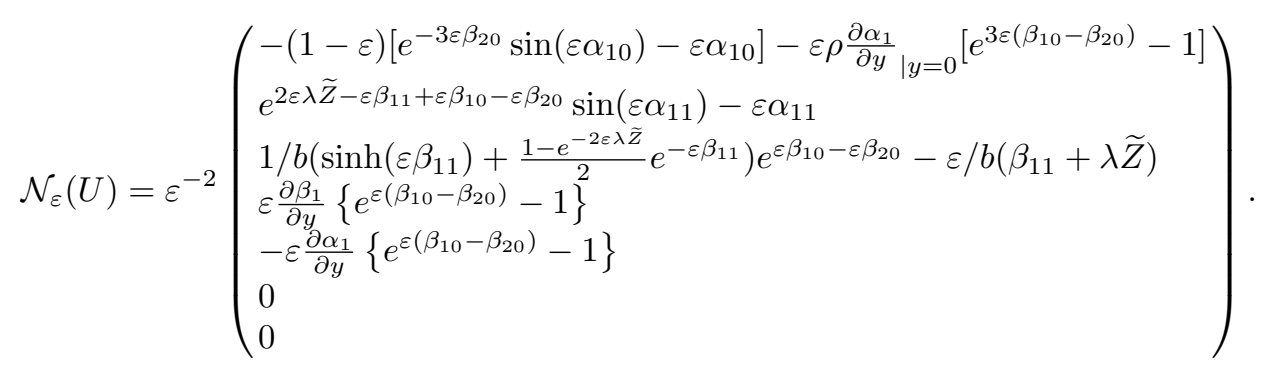

We observe that the two last components of $\mathcal{N}_{\varepsilon}(U)$ are zero, which allows to define $\mathcal{N}_{\varepsilon}$ even for $U$ with non differentiable components $\alpha_{2}, \beta_{2}$ (this is used in the definition of the forthcoming space $\widehat{\mathbb{D}}$ ).

In this example, notice that we have the following combination of the Bernoulli first integrals (hence independent of $x$ ), well defined in $\mathbb{H}$, which reads

$$
\begin{aligned}
h= & (1-\varepsilon)\left\{-\frac{1}{2 \lambda} e^{-2 \lambda \varepsilon \widetilde{Z}}-\int_{0}^{1}\left(e^{-\varepsilon \beta_{1}} \cos \left(\varepsilon \alpha_{1}\right)-1\right) d y\right\} \\
& +\frac{1}{2} e^{2 \varepsilon \beta_{20}}-\frac{\rho}{2} e^{2 \varepsilon \beta_{10}} .
\end{aligned}
$$

\section{Characterization of the Spectrum}

Let us now study the reversible system (1.1) for $\varepsilon>0$. We first need a scaling in the coordinates and in the variables which dilates the spectrum of the linear operator $L_{\varepsilon}$ with a factor $1 / \varepsilon$. This scaling is explicitly given in the examples of section 2 . The equation (1.1) now reads

$$
\frac{d U}{d x}=\mathcal{L}_{\varepsilon} U+\mathcal{N}_{\varepsilon}(U),
$$

which is the reversible system we want to study. We denote by $S$ the reversibility symmetry: $S$ anticommutes with $\mathcal{L}_{\varepsilon}$ and $\mathcal{N}_{\varepsilon}$

$$
\begin{aligned}
\mathcal{L}_{\varepsilon} S U & =-S \mathcal{L}_{\varepsilon}, \\
\mathcal{N}_{\varepsilon}(S U) & =-S \mathcal{N}_{\varepsilon}(U) .
\end{aligned}
$$


In finite dimension we only need to know the eigenvalues of the linear operator $\mathcal{L}_{\varepsilon}$ to describe the dynamics of such systems. In the present paper this description is not sufficient because of the presence of the essential spectrum and the singularities due to this part of the spectrum are unknown. Therefore we make several assumptions on the resolvent to describe the structure of the spectrum near the origin as $\varepsilon$ tends towards zero. We need three assumptions and we illustrate each of them on the detailed example of section 2 .

\subsection{Working spaces and notations}

Before giving the assumptions on the resolvent operator we have to introduce some spaces. We first recall that $\mathbb{H}$ is the basic space for (3.1) and that $\mathbb{D}$ is the domain of the linear operator $\mathcal{L}_{\varepsilon}$. We now introduce the Banach spaces

$$
\begin{aligned}
& \widetilde{\mathbb{H}} \subset \mathbb{H}, \\
& \widetilde{\mathbb{D}} \subset \mathbb{D},
\end{aligned}
$$

where $\widetilde{\mathbb{H}}$ is dense in $\mathbb{H}$ and $\widetilde{\mathbb{D}}$ is dense in $\mathbb{D}$ and such that $\mathcal{L}_{\varepsilon}: \widetilde{\mathbb{D}} \mapsto \widetilde{\mathbb{H}}$ (we describe below the spaces $\widetilde{\mathbb{H}}$ and $\widetilde{\mathbb{D}}$ for the examples). Actually, $\mathcal{L}_{\varepsilon}$ turns out to be Fredholm in $\widetilde{\mathbb{H}}$ for our examples. Introducing $\widetilde{\mathbb{H}}$ is necessary for describing the smoothness in $k$ near 0 of some important $k$ dependent operators occurring in the detailed form of the resolvent $\left(i k-\mathcal{L}_{\varepsilon}\right)^{-1}$ explicitly given in the forthcoming hypothesis $\mathrm{H} 1$.

Notice that we choose to work in a space $\mathbb{H}$ where $\mathcal{L}_{\varepsilon}$ is not Fredholm. This is due to the fact that a non trivial solution $U$ decaying as $|x| \rightarrow \infty$ does not necessarily lead to $U(x)$ in $\widetilde{\mathbb{H}}$. In our examples, this results from the fact that if one imposes a decay in $1 / x^{2}$ as $|x| \rightarrow \infty$ for a harmonic function in the half space $y<0$, then one cannot also impose an exponential decay in $y$ as $y \rightarrow-\infty$. The decays in $x$ and $y$ are linked.

We also need a Banach space $\widehat{\mathbb{D}}$

$$
\mathbb{D} \subset \widehat{\mathbb{D}} \subset \mathbb{H},
$$

which is used in the description of the regularity of the resolvent operator and this space is important in the nonlinear study. The resolvent operator $\left(i k-\mathcal{L}_{\varepsilon}\right)^{-1}$ considered in $\mathcal{L}(\widetilde{\mathbb{H}}, \widehat{\mathbb{D}})$ is a smooth function of $k$ in $\mathbb{R} \backslash\{0\}$ (see assumptions H1, H2 and H3). At last, $\widetilde{\mathbb{H}}$ and $\widehat{\mathbb{D}}$ must be chosen so that

$$
\mathcal{N}_{\varepsilon}: \widehat{\mathbb{D}} \rightarrow \widetilde{\mathbb{H}} \text {. }
$$

For our examples this property is related to the fact that the components of $\mathcal{N}_{\varepsilon}$ corresponding to the domain $y<0$ (two last components) cancel. Since, precisely these components are source of complications, the above property simplifies our study.

Example. For the example detailed in section 2, the Banach spaces $\widetilde{\mathbb{H}}, \widetilde{\mathbb{D}}$ and $\widehat{\mathbb{D}}$ are explicitly given below:

$$
\begin{aligned}
\widetilde{\mathbb{H}} & =\mathbb{R}^{3} \times\left\{\mathcal{C}^{0}(0,1)\right\}^{2} \times\left\{\mathcal{C}_{\varepsilon}^{\exp }\left(\mathbb{R}^{-}\right)\right\}^{2}, \\
\widetilde{\mathbb{D}} & =\mathbb{R}^{3} \times\left\{\mathcal{C}^{1}(0,1)\right\}^{2} \times\left\{\mathcal{C}_{\varepsilon}^{1, \exp }\left(\mathbb{R}^{-}\right)\right\}^{2},
\end{aligned}
$$


where $\mathcal{C}_{\varepsilon}^{\exp }\left(\mathbb{R}^{-}\right)=\left\{f \in \mathcal{C}^{0}\left(\mathbb{R}^{-}\right),\|f\|_{\varepsilon}^{\exp }<\infty\right\}$ with the norm

$$
\|f\|_{\varepsilon}^{\exp }=\sup _{y \in \mathbb{R}^{-}}|f(y)| e^{-\lambda y / 2 \varepsilon},
$$

and $\mathcal{C}_{\varepsilon}^{1, \exp }\left(\mathbb{R}^{-}\right)=\left\{f \in \mathcal{C}_{\varepsilon}^{\exp }\left(\mathbb{R}^{-}\right), f^{\prime} \in \mathcal{C}_{\varepsilon}^{\exp }\left(\mathbb{R}^{-}\right)\right\}$with the norm

$$
\|f\|_{\varepsilon}^{1, \exp }=\varepsilon\left\|f^{\prime}\right\|_{\varepsilon}^{\exp }+\|f\|_{\varepsilon}^{\exp } .
$$

Then $\mathcal{L}_{\varepsilon}$ considered in $\mathcal{L}(\widetilde{\mathbb{H}})$ is Fredholm on the real axis, one can show that the only eigenvalue is 0 , and for any $\lambda$ real, $\lambda \mathbb{I}-\mathcal{L}_{\varepsilon}$ has a closed range, of codimension 1 if $\lambda \neq 0$ and 2 if $\lambda=0$. Note that we choose spaces for which the components of the lower fluid have an exponential decay rate.

We now observe that we don't need the differentiability of $\alpha_{2}$ and $\beta_{2}$ (i.e. the components of the lower fluid) to define $\mathcal{N}_{\varepsilon}(U)$, hence $\mathcal{N}_{\varepsilon}(U)$ is well-defined for $U \in$ $\widehat{\mathbb{D}} \supset \mathbb{D}$

$$
\begin{aligned}
\widehat{\mathbb{D}}= & \mathbb{R}^{3} \times\left\{\mathcal{C}^{1}(0,1)\right\}^{2} \times\left\{\mathcal{C}_{1}^{0}\left(\mathbb{R}^{-}\right)\right\}^{2} \\
& \cap\left\{\alpha_{1}(0)=\alpha_{2}(0), \alpha_{11}=\alpha_{1}(1), \beta_{20}=\beta_{2}(0)\right\} .
\end{aligned}
$$

Notice that $\mathcal{N}_{\varepsilon}$ maps $\widehat{\mathbb{D}}$ in $\widetilde{\mathbb{H}} \subset \mathbb{H}$ since its two last components are zero

Definition For a Banach space $\mathbb{E}$ we define the following function spaces

$$
\begin{aligned}
\mathcal{C}_{\lim }^{p}(\mathbb{R}, \mathbb{E})= & \left\{f: \mathbb{R} \rightarrow \mathbb{E} ; f \in \mathcal{C}^{p}(\mathbb{R} \backslash\{0\}), f \text { continuous in } 0\right. \text { and } \\
& \left.f^{(n)} \text { has limits in } 0^{ \pm} \forall n \leq p\right\},
\end{aligned}
$$

with the sup norms on the $p$ first derivatives. We also introduce the following $\varepsilon-$ dependent spaces

$$
\mathcal{C}_{\lim , \varepsilon}^{p}(\mathbb{R}, \mathbb{E})=\mathcal{C}_{\lim }^{p}(\mathbb{R}, \mathbb{E}) \cap\left\{\max _{x \in \mathbb{R}}\left\|f^{(n)}(x)\right\|_{\mathbb{E}} \leq c \varepsilon^{n}, n \leq p\right\} .
$$

For a Banach space $\mathbb{E}$ we denote by $\mathbb{E}^{*}$ the Banach space of bounded linear forms on $\mathbb{E}$. We use the notation $\mathcal{F}(f)$ or $\hat{f}$ for the Fourier transform of a function $f$ and the inverse transform is denoted by $\mathcal{F}^{-1}$. The Hilbert transform $\mathcal{H}(f)$ of a function $f$ is defined by

$$
\mathcal{H}(f)(x)=p \cdot v \cdot \int_{\mathbb{R}} \frac{f(s)}{x-s} d s .
$$

We recall that

$$
\mathcal{F}(\mathcal{H}(f))=-i \operatorname{sgn}(k) \hat{f}
$$

\subsection{Assumptions on the linearized operator}

\subsubsection{Hypothesis H1}

We now state the main assumption on the linear operator. This assumption gives the structure of the resolvent operator near the origin on the imaginary axis, and describes partly the spectrum of $\mathcal{L}_{\varepsilon}$, obtained after the rescaling which dilates the spectrum of $L_{\varepsilon}$ as explicitly explained in the two examples. 
Hypothesis H1 (resolvent operator for small $\varepsilon|k|$ ) There exists $\delta>0$ such that for $k \in \mathbb{R} \backslash\{0\}, \varepsilon|k| \leq \delta$ and $V \in \mathbb{H}$, the resolvent operator is decomposed as follows

$$
\left(i k-\mathcal{L}_{\varepsilon}\right)^{-1} V=\frac{\xi_{\varepsilon, k}^{*}(V)}{i k \varepsilon \Delta} \xi_{0}+\frac{\eta_{\varepsilon, k}^{*}(V)}{\Delta} \theta_{k}+\varepsilon S_{\varepsilon, k}(V),
$$

with the properties described below in four parts.

\section{Hypothesis H1a: On the dispersion equation.}

Consider the dispersion equation, i.e. the equation obtained when one is looking for non trivial solutions of the linear system

$$
\left(i k-\mathcal{L}_{\varepsilon}\right) U=0, \quad k \in \mathbb{C}, \quad U \in \mathbb{D} .
$$

Then, for $k \in \mathbb{R} \backslash\{0\}$, and near 0 this equation takes the form

$$
i k \varepsilon \Delta(\varepsilon, k)=0
$$

where the map $k \mapsto \Delta(\varepsilon, k) \in \mathcal{C}_{\lim }^{3}(\mathbb{R}, \mathbb{R})$ is even and satisfies

$$
\begin{aligned}
\Delta(\varepsilon, k) & =1+a|k|+O\left(\varepsilon k^{2}\right), a>0 \\
\Delta^{(3)}\left(\varepsilon, 0^{ \pm}\right) & =O\left(\varepsilon^{2}\right) .
\end{aligned}
$$

Remark 1a. We recognize the root $k=0$ of the dispersion equation, due to the simple eigenvalue in 0 for the operator $\mathcal{L}_{\varepsilon}$. The absolute value of $k$ in $\Delta$ shows that $\Delta$ is not analytic, and this is related to the fact that 0 lies in the essential spectrum of $\mathcal{L}_{\varepsilon}$. The evenness comes from the reversibility of the system.

Hypothesis H1b: On the splitting of the space and the projection associated with the kernel of $\mathcal{L}_{\varepsilon}$.

The vector $\xi_{0} \in \widetilde{\mathbb{D}}$ is symmetric $\left(S \xi_{0}=\xi_{0}\right)$ and satisfies $\mathcal{L}_{\varepsilon} \xi_{0}=0$. Moreover the kernel of $\mathcal{L}_{\varepsilon}$ is one-dimensional.

There exists $p_{0}^{*} \in \mathbb{H}^{*}$ such that $p_{0}^{*}(S V)=p_{0}^{*}(V), p_{0}^{*}\left(\xi_{0}\right)=1$ and $p_{0}^{*}\left(\mathcal{L}_{\varepsilon} V\right): \mathbb{D} \rightarrow \mathbb{R}$ can be extended as a continuous map: $\widehat{\mathbb{D}} \rightarrow \mathbb{R}$.

There exists $\delta>0$ such that for $k \in \mathbb{R} \backslash\{0\}, \varepsilon|k| \leq \delta$ and $V \in \mathbb{H}$, we have

$$
p_{0}^{*}\left(i k-\mathcal{L}_{\varepsilon}\right)^{-1} V=\frac{\xi_{\varepsilon, k}^{*}(V)}{i k \varepsilon \Delta(\varepsilon, k)},
$$

where, for $k \neq 0, \xi_{\varepsilon, k}^{*} \in \mathbb{H}^{*}$, and the map $k \mapsto \xi_{\varepsilon, k}^{*} \in \mathcal{C}_{\lim , \varepsilon}^{3}\left(\mathbb{R}, \widetilde{\mathbb{H}}^{*}\right)$ is such that we can define the forms $\chi_{\varepsilon}^{*}$ and $\zeta_{\varepsilon, k}^{*}$ with

$$
\begin{aligned}
\xi_{\varepsilon, k}^{*} & =\xi_{\varepsilon}^{*}+\varepsilon|k| \chi_{\varepsilon}^{*}+\zeta_{\varepsilon, k}^{*}, \\
\xi_{\varepsilon}^{*} & \in \mathbb{H}^{*}, \quad \chi_{\varepsilon}^{*} \in \widetilde{\mathbb{H}}^{*}, \quad \zeta_{\varepsilon, 0}^{*}=0, \\
k & \mapsto \zeta_{\varepsilon, k}^{*} \in \mathcal{C}^{1}\left(\mathbb{R}, \widetilde{\mathbb{H}}^{*}\right) \cap \mathcal{C}_{\lim , \varepsilon}^{3}\left(\mathbb{R}, \widetilde{\mathbb{H}}^{*}\right) .
\end{aligned}
$$

Remark 1b. For splitting the space $\mathbb{H}$, we use the form $p_{0}^{*}$ which is independent of $\varepsilon$. A more natural choice would be the form $\xi_{\varepsilon}^{*}$, however this would lead to difficulties because of its singular behavior when $\varepsilon \rightarrow 0$ : notice that the map $V \mapsto \frac{\xi_{\varepsilon}^{*}(V)}{\varepsilon} \xi_{0}$ is the 
projector commuting with $\mathcal{L}_{\varepsilon}$ (see Lemma 3.1), associated with the 0 eigenvalue, and $\xi_{\varepsilon}^{*}\left(\xi_{0}\right)=\varepsilon$. Notice also that we put in $\chi_{\varepsilon}^{*}$ the jump of the derivative of $k \mapsto \xi_{\varepsilon, k}^{*}$ at $k=0^{ \pm}$.

Hypothesis H1c: Specific assumption on the singularity related to the essential spectrum in 0.

The singular part of $\left(i k-\mathcal{L}_{\varepsilon}\right)^{-1} V$ in $\operatorname{ker} p_{0}^{*}$ reads

$$
\frac{\eta_{\varepsilon, k}^{*}(V)}{\Delta(\varepsilon, k)} \theta_{k},
$$

where the map $k \mapsto \theta_{k}$ is $\mathcal{C}^{0}(\mathbb{R} \backslash\{0\}, \mathbb{D}), k \theta_{k}$ is bounded in $\mathbb{D}$, and $S \theta_{k}=-\theta_{-k}$, $p_{0}^{*}\left(\theta_{k}\right)=0$, and

$$
\xi_{\varepsilon}^{*}\left(\theta_{k}\right)=i \operatorname{sgn}(k) .
$$

For $k \neq 0, \eta_{\varepsilon, k}^{*} \in \mathbb{H}^{*}$, and the map $k \mapsto \eta_{\varepsilon, k}^{*} \in \mathcal{C}_{\lim , \varepsilon}^{3}\left(\mathbb{R}, \widetilde{\mathbb{H}}^{*}\right)$ takes the form

$$
\eta_{\varepsilon, k}^{*}=\eta_{\varepsilon}^{*}+\beta_{\varepsilon, k}^{*}, \quad \beta_{\varepsilon, 0}^{*}=0, \quad \eta_{\varepsilon}^{*} \in \widetilde{\mathbb{H}}^{*}, \beta_{\varepsilon, k}^{*} \in \mathcal{C}^{1}\left(\mathbb{R}, \widetilde{\mathbb{H}}^{*}\right)
$$

Remark 1c. The new vector $\theta_{k}$ is not continuous in $k=0$ (because of the presence of the essential spectrum). The form of its discontinuity is not explicitly written here, and more precisions are given in Hypothesis H2. We notice below in lemma 3.1 that assumption (3.4) is very weak.

Hypothesis H1d: Regular part of the resolvent near 0 for $k \in \mathbb{R} \backslash\{0\}$.

The regular part of $\left(i k-\mathcal{L}_{\varepsilon}\right)^{-1} V$ reads

$$
\varepsilon S_{\varepsilon, k}(V)
$$

where $S_{\varepsilon, k}(V) \in \mathcal{L}(\mathbb{H}, \mathbb{D})$ for $k \neq 0$, and the map $k \mapsto S_{\varepsilon, k} \in \mathcal{C}_{\lim , \varepsilon}^{3}(\mathbb{R}, \mathcal{L}(\widetilde{\mathbb{H}}, \widehat{\mathbb{D}}))$, with $p_{0}^{*}\left(S_{\varepsilon, k}(V)\right)=0$.

Remark 1. The decomposition (3.3) is also valid in the reversible finite dimensional case, when a pair of imaginary eigenvalues exists for $\varepsilon<0$, collides in 0 for $\varepsilon=0$, and becomes a real pair of symmetric eigenvalues for $\varepsilon>0$. However in such a case $\Delta$ is analytic in $k$ near 0 , and the linear form $\xi_{\varepsilon, k}^{*}$ is a smooth function of $k$ in $\mathbb{H}^{*}$.

Example. Let us check Hypothesis H1 on the example shown in section 2 (for the other example the proof is similar). The computations are straightforward, and are mostly in [5]. We obtain $\Delta$ in $\mathcal{C}_{\lim }^{\infty}(\mathbb{R}, \mathbb{R})$ with

$$
\Delta(\varepsilon, k)=1+a|k|+O\left(\varepsilon k^{2}\right), a=\lambda^{-1}(\lambda-1) .
$$

We choose the linear form $p_{0}^{*}$ defined by

$$
p_{0}^{*}(U)=-\beta_{1}(1) \quad\left(=-\beta_{11}\right),
$$

and vectors $\xi_{0}$ and $\theta_{k}$ by

$$
\xi_{0}=\left(0, \lambda^{-1}, 0,0,-1,0,0\right)^{t}
$$




$$
\theta_{k}=\left(i \operatorname{sgn}(k), 0, \frac{-1}{\lambda-1}, 1-\frac{\lambda}{\lambda-1} y, 0, e^{|k| y}, i \operatorname{sgn}(k) e^{|k| y}\right)^{t} .
$$

We finally have for $V=\left(a_{1}, a_{2}, a_{3}, f_{1}, g_{1}, f_{2}, g_{2}\right)^{t} \in \widetilde{\mathbb{H}}$

$$
\begin{aligned}
& \xi_{\varepsilon}^{*}(V)=a_{1}-\rho g_{10}+(1-\varepsilon) a_{2}+(1-\varepsilon) \int_{0}^{1} g_{1}(\tau) d \tau, \text { also defined in } \mathbb{H}, \\
& \chi_{\varepsilon}^{*}(V)=-a_{2}-\int_{0}^{1} g_{1}(\tau) d \tau+\varepsilon^{-1} \int_{-\infty}^{0} g_{2}(\tau) d \tau, \\
& \eta_{\varepsilon}^{*}(V)=\lambda^{-1}(1-\lambda)\left(a_{1}-\rho g_{10}\right)-\rho a_{2}-\rho \int_{0}^{1} g_{1}(\tau) d \tau+\int_{-\infty}^{0} g_{2}(\tau) d \tau,
\end{aligned}
$$

and the linear relation between these three forms is

$$
\eta_{\varepsilon}^{*}=-\frac{\lambda-1}{\lambda} \xi_{\varepsilon}^{*}+\varepsilon \chi_{\varepsilon}^{*},
$$

and we prove in the next lemma that this relation is equivalent to the assumption $\xi_{\varepsilon}^{*}\left(\theta_{k}\right)=i \operatorname{sgn}(k)$. We observe that the linear forms $\chi_{\varepsilon}^{*}$ and $\eta_{\varepsilon}^{*}$ cannot be defined in $\mathbb{H}$ because of an insufficient decay rate of the component $g_{2}$ in the lower layer. The exponential decay rate in $\widetilde{\mathbb{H}}$ provides convergence and boundedness with respect to $\varepsilon$ of the integrals.

\subsubsection{First properties}

Hypothesis $\mathrm{H} 1$ states the main assumptions on the resolvent, except an additional further precision on $\theta_{k}$ (see hypothesis H2). Next Lemma gives some properties on the linear forms, which are consequences of hypothesis H1, and which clarify the relationship (3.4) and the characterization of the closure of the range of $\mathcal{L}_{\varepsilon}$.

Lemma 3.1. If $\mathcal{L}_{\varepsilon}$ satisfies the Hypothesis H1, without (3.4), then the following properties hold

i) Symmetry results

$$
\begin{aligned}
\xi_{\varepsilon, k}^{*}(S V) & =\xi_{\varepsilon,-k}^{*}(V), \eta_{\varepsilon, k}^{*}(S V)=\eta_{\varepsilon,-k}^{*}(V), S S_{\varepsilon, k}(V)=-S_{\varepsilon,-k}(-V), \\
\xi_{\varepsilon}^{*}(S V) & =\xi_{\varepsilon}^{*}(V), \quad \eta_{\varepsilon}^{*}(S V)=\eta_{\varepsilon}^{*}(V), \chi_{\varepsilon}^{*}(S V)=\chi_{\varepsilon}^{*}(V) .
\end{aligned}
$$

ii)

$$
\begin{aligned}
\xi_{\varepsilon}^{*}\left(\xi_{0}\right) & =\varepsilon, \quad \chi_{\varepsilon}^{*}\left(\xi_{0}\right)=a \\
\xi_{\varepsilon}^{*}\left(\mathcal{L}_{\varepsilon} U\right) & =0, \quad \forall U \in \mathbb{D}, \quad \chi_{\varepsilon}^{*}\left(\mathcal{L}_{\varepsilon} U\right)=0, \forall U \in \widetilde{\mathbb{D}} .
\end{aligned}
$$

iii) There is $\alpha \in \mathbb{C}$ such that $\xi_{\varepsilon}^{*}\left(\theta_{k}\right)=i \alpha \operatorname{sgn}(k)+o(1) \quad\left(k \rightarrow 0^{ \pm}\right)$, and $a \xi_{\varepsilon}^{*}=$ $\varepsilon \chi_{\varepsilon}^{*}-\alpha \eta_{\varepsilon}^{*}$, and the closure of the range of $\mathcal{L}_{\varepsilon}$ has codimension 2.

Remark. We observe that if we make $k \rightarrow 0$ in $\mathrm{H} 1$ then we have a limit in (3.3) provided that $V \in \widetilde{\mathbb{H}}$ satisfies

$$
\xi_{\varepsilon}^{*}(V)=\chi_{\varepsilon}^{*}(V)=\eta_{\varepsilon}^{*}(V)=0 .
$$


Hence, these are sufficient conditions for $V$ to be in the range of $\mathcal{L}_{\varepsilon}$. What we prove in lemma $3.1 \mathrm{iii}$ ) is that the closure of the range of $\mathcal{L}_{\varepsilon}$ has codimension at most 2, since $\operatorname{ker} \xi_{\varepsilon}^{*} \cap \operatorname{ker} \eta_{\varepsilon}^{*} \cap \operatorname{ker} \chi_{\varepsilon}^{*}$ has dimension 2 and $\widetilde{\mathbb{H}}$ is dense in $\mathbb{H}$.

We notice that the assumption (3.4) in Hypothesis H1 is equivalent to assume that $\alpha$ is non zero in Lemma 3.1 iii).

\section{Proof of lemma 3.1.}

i) results from $\mathcal{L}_{\varepsilon} S=-S \mathcal{L}_{\varepsilon}$ and Hypothesis H1.

To prove ii) we use $\left(i k-\mathcal{L}_{\varepsilon}\right)^{-1} \xi_{0}=\frac{1}{i k} \xi_{0}$ and apply $p_{0}^{*}$ to (3.3). This leads to

$$
\xi_{\varepsilon, k}^{*}\left(\xi_{0}\right)=\varepsilon \Delta=\varepsilon\left(1+a|k|+O\left(\varepsilon k^{2}\right)\right),
$$

which gives $\xi_{\varepsilon}^{*}\left(\xi_{0}\right)=\varepsilon, \chi_{\varepsilon}^{*}\left(\xi_{0}\right)=a$ and $\zeta_{\varepsilon, k}^{*}\left(\xi_{0}\right)=O\left(\varepsilon^{2} k^{2}\right)$.

To prove the second part of ii) we use hypothesis H1 with $V=\left(i k-\mathcal{L}_{\varepsilon}\right) U$ for any $U \in \widetilde{\mathbb{D}}$, again applying $p_{0}^{*}$ :

$$
\varepsilon \Delta p_{0}^{*}(U)=\xi_{\varepsilon, k}^{*}(U)-\frac{\xi_{\varepsilon, k}^{*}\left(\mathcal{L}_{\varepsilon} U\right)}{i k} .
$$

Taking the limit when $k \rightarrow 0$ we obtain $\xi_{\varepsilon}^{*}\left(\mathcal{L}_{\varepsilon} U\right)=0$ by continuity of $k \xi_{\varepsilon, k}^{*}$ and $\xi_{\varepsilon}^{*}\left(\mathcal{L}_{\varepsilon} U\right)=0$ for all $U \in \mathbb{D}$ thanks to the density of $\widetilde{\mathbb{D}} \subset \mathbb{D}$. Now with $U \in \widetilde{\mathbb{D}}$ we have $\mathcal{L}_{\varepsilon} U \in \widetilde{\mathbb{H}}$ and with the same proof $\chi_{\varepsilon}^{*}\left(\mathcal{L}_{\varepsilon} U\right)=0$.

We also have

$$
\xi_{\varepsilon}^{*}(V)=\xi_{\varepsilon}^{*}\left(\left(i k-\mathcal{L}_{\varepsilon}\right) U\right)=i k \xi_{\varepsilon}^{*}(U),
$$

then applying $\xi_{\varepsilon}^{*}$ to $(3.3)$

$$
\frac{1}{k}\left(\Delta \xi_{\varepsilon}^{*}(V)-\xi_{\varepsilon, k}^{*}(V)\right)=i \eta_{\varepsilon, k}^{*}(V) \xi_{\varepsilon}^{*}\left(\theta_{k}\right)+i \varepsilon \Delta \xi_{\varepsilon}^{*}\left(S_{\varepsilon, k}(V)\right),
$$

it results that $\xi_{\varepsilon}^{*}\left(\theta_{k}\right)$ has limits when $k \rightarrow 0^{ \pm}$. Therefore computing the jump of $\xi_{\varepsilon}^{*}\left(\theta_{k}\right)$ when $k \rightarrow 0^{ \pm}$, we obtain

$$
a \xi_{\varepsilon}^{*}(V)=\varepsilon \chi_{\varepsilon}^{*}(V)+\eta_{\varepsilon}^{*}(V) \frac{1}{2}\left[i \xi_{\varepsilon}^{*}\left(\theta_{k}\right)\right]_{0^{-}}^{0^{+}} .
$$

Since $\xi_{\varepsilon}^{*}\left(\theta_{-k}\right)=\xi_{\varepsilon}^{*}\left(-S \theta_{k}\right)=-\xi_{\varepsilon}^{*}\left(\theta_{k}\right)$

$$
\lim _{0^{+}} \xi_{\varepsilon}^{*}\left(\theta_{k}\right)=-\lim _{0^{-}} \xi_{\varepsilon}^{*}\left(\theta_{k}\right)
$$

and the number

$$
\alpha=-\lim _{k \rightarrow 0} i \operatorname{sgn}(k) \xi_{\varepsilon}^{*}\left(\theta_{k}\right),
$$

is well-defined. This allows us to write

$$
a \xi_{\varepsilon}^{*}=\varepsilon \chi_{\varepsilon}^{*}-\alpha \eta_{\varepsilon}^{*},
$$

and lemma 3.1 is proved.

Remark. Since we assume in H1 that $\xi_{\varepsilon}^{*}\left(\theta_{k}\right)=i \operatorname{sgn}(k)$ then automatically $\alpha=1$ in Lemma 3.1. Therefore the relation

$$
a \xi_{\varepsilon}^{*}=\varepsilon \chi_{\varepsilon}^{*}-\eta_{\varepsilon}^{*}
$$

holds. 


\subsubsection{Spaces for the $x$ - dependence.}

We introduce here the Banach spaces which describe the dependence in $x$ of the solutions of our system. Let $\mathbb{E}$ be a Banach space and $0<\alpha<1$, then we define the following Hölder space

$$
\begin{aligned}
& B_{p}^{\alpha}(\mathbb{E})=\left\{f \in \mathcal{C}^{\alpha}(\mathbb{R}, \mathbb{E}) ;\|f\|_{p, \mathbb{E}}^{\alpha}<\infty\right\}, \\
& \|f\|_{p, \mathbb{E}}^{\alpha}=\sup _{x \in \mathbb{R}}\left(1+|x|^{p}\right)\|f(x)\|_{\mathbb{E}}+\sup _{x \in \mathbb{R}, 0<|\delta| \leq 1}\left(1+|x|^{p}\right) \frac{\|f(x+\delta)-f(x)\|_{\mathbb{E}}}{|\delta|^{\alpha}} .
\end{aligned}
$$

We also need $B_{2}^{1, \alpha}(\mathbb{R})$ defined by

$$
B_{2}^{1, \alpha}(\mathbb{R})=\left\{f \in B_{2}^{\alpha}(\mathbb{R}) ; \frac{d f}{d x} \in B_{2}^{\alpha}(\mathbb{R})\right\},
$$

with the corresponding norm denoted by $\|f\|_{2, \mathbb{R}}^{1, \alpha}$.

To make precise the behavior of $\theta_{k}$ in the neighborhood of 0 we need to introduce a new Banach space. This space is also important in the construction of the solutions of the equation because this construction strongly depends on the structure of the vector $\theta_{k}$. As it is seen in (3.5) for our example, $\theta_{k}$ is not defined in $\mathbb{H}$ for $k=0$.

We introduce a Banach space $\mathcal{B}^{\alpha}(\widehat{\mathbb{D}})$ such that

$$
\mathcal{B}^{\alpha}(\widehat{\mathbb{D}}) \supset B_{2}^{\alpha}(\widehat{\mathbb{D}}),
$$

with the associated norm \|\|$_{\mathcal{B}}$, and its main properties are indicated in the following hypothesis $\mathrm{H} 2$. We assume that for $U \in \mathcal{B}^{\alpha}(\widehat{\mathbb{D}})$ and any $x \in \mathbb{R}$ then $U(x) \in \widehat{\mathbb{D}}$. Actually this space is a little larger than $B_{2}^{\alpha}(\widehat{\mathbb{D}})$ (see example below). One of the main assumption on $\mathcal{B}^{\alpha}(\widehat{\mathbb{D}})$ is the following hypothesis, which gives the link between $\mathcal{B}^{\alpha}(\widehat{\mathbb{D}})$ and the vector $\theta_{k}$

\section{Hypothesis H2: Characterization of $\boldsymbol{\theta}_{k}$}

H2a "regularity" of $\boldsymbol{i k} \boldsymbol{\theta}_{k}$

For any $u \in B_{2}^{1, \alpha}(\mathbb{R})$ we define the linear map $\mathcal{T}$ by

$$
u \mapsto \mathcal{T}(u)=\mathcal{F}^{-1}\left(-i k \hat{u} \theta_{k}\right) ;
$$

$\mathcal{T}$ is bounded from $B_{2}^{1, \alpha}(\mathbb{R})$ into $\mathcal{B}^{\alpha}(\widehat{\mathbb{D}})$.

H2b The linear forms $\xi_{\varepsilon}^{*}$ and $p_{0}^{*} \mathcal{L}_{\varepsilon}$ are continuous from $\mathcal{B}^{\alpha}(\widehat{\mathbb{D}})$ to $B_{2}^{\alpha}(\mathbb{R})$

$$
\begin{aligned}
\xi_{\varepsilon}^{*} & \in \mathcal{L}\left(\mathcal{B}^{\alpha}(\widehat{\mathbb{D}}), B_{2}^{\alpha}(\mathbb{R})\right), \\
p_{0}^{*} \mathcal{L}_{\varepsilon} & \in \mathcal{L}\left(\mathcal{B}^{\alpha}(\widehat{\mathbb{D}}), B_{2}^{\alpha}(\mathbb{R})\right) .
\end{aligned}
$$

Remark 2b. From Hypothesis H1b, we know that $\xi_{\varepsilon}^{*}$ and $p_{0}^{*} \mathcal{L}_{\varepsilon}$ are in $\mathcal{L}\left(B_{2}^{\alpha}(\widehat{\mathbb{D}}), B_{2}^{\alpha}(\mathbb{R})\right)$. The above assumption extends these forms to $\mathcal{B}^{\alpha}(\widehat{\mathbb{D}})$ instead of $B_{2}^{\alpha}(\widehat{\mathbb{D}})$.

Remark 2. A consequence of (3.4) and Hypothesis H2 is

$$
\xi_{\varepsilon}^{*}(\mathcal{T}(u))=\mathcal{H}\left(\frac{d u}{d x}\right)
$$

so, we can understand Hypothesis $\mathrm{H} 2$ in considering the convolution by $\mathcal{F}^{-1}\left(\theta_{k}\right)$ as a sort of "generalized" Hilbert transform. 
Example. Our detailed example (two superposed fluid layers, bottom one infinitely deep, strong surface tension at the free surface) leads to the following space

$$
\begin{aligned}
\mathcal{B}^{\alpha}(\widehat{\mathbb{D}})= & \left\{U=\left(\beta_{20}, \widetilde{Z}, \alpha_{11}, \alpha_{1}, \beta_{1}, \alpha_{2}, \beta_{2}\right)^{t}, \beta_{20}(x)=\beta_{2}(x, 0), \alpha_{1}(x, 1)=\alpha_{11}(x),\right. \\
& \left.\alpha_{1}(x, 0)=\alpha_{2}(x, 0), \alpha_{1}, \beta_{1} \in B_{2}^{\alpha}\left(\mathcal{C}^{1}(0,1)\right), \alpha_{2}, \beta_{2} \in B_{\alpha}^{-}\right\},
\end{aligned}
$$

where

$$
\begin{aligned}
& B_{\alpha}^{-}=\left\{(x, y) \mapsto f(x, y): \mathbb{R} \times \mathbb{R}^{-} \rightarrow \mathbb{R}, f \text { is } \mathcal{C}^{0} \text { in } y, \mathcal{C}^{\alpha} \text { in } x,\|f\|_{B_{\alpha}^{-}}<\infty\right\}, \\
&\|f\|_{B_{\alpha}^{-}}= \sup _{x \in \mathbb{R}, y<0} \frac{1+|x|^{2}+|y|^{2}}{1+|y|}|f(x, y)|+ \\
&+\sup _{x \in \mathbb{R}, y<0,0<|\delta| \leq 1} \frac{1+|x|^{2}+|y|^{2}}{1+|y|} \frac{|f(x+\delta, y)-f(x, y)|}{|\delta|^{\alpha}} .
\end{aligned}
$$

Note that $\mathcal{B}^{\alpha}(\widehat{\mathbb{D}})$ differs from $B_{2}^{\alpha}(\widehat{\mathbb{D}})$ only in the components $\left(\alpha_{2}, \beta_{2}\right)$ of the lower layer. For these components in the definition of $B_{\alpha}^{-}$the decay rate in the horizontal variable $x$ and the decay rate in the vertical variable $y$ are closely linked and $\alpha_{2}, \beta_{2}$ are only continuous functions of $y$ whereas they would be $\mathcal{C}^{1}$ for $U \in B_{2}^{\alpha}(\mathbb{D})$.

We remark that the definitions of $\xi_{\varepsilon}^{*}(V)$ and $p_{0}^{*}\left(\mathcal{L}_{\varepsilon} V\right)=\left.\frac{1}{\varepsilon} \frac{\partial \alpha_{1}}{\partial y}\right|_{\mid y=1}$ only involve the components $\beta_{20}, \widetilde{Z}, \alpha_{1}$ and $\beta_{1}$ of $V$ then Hypothesis H2b is satisfied.

If $u \in B_{2}^{1, \alpha}(\mathbb{R})$ then

$$
\mathcal{T}(u)=\mathcal{F}^{-1}\left(-i k \hat{u} \theta_{k}\right)=\left(\beta_{20}, 0, \alpha_{11}, \alpha_{1}, 0, \alpha_{2}, \beta_{2}\right)^{t},
$$

where

$$
\begin{aligned}
\beta_{20} & =\mathcal{H}\left(u^{\prime}\right), \alpha_{1}=\left(\frac{\lambda}{\lambda-1} y-1\right) u^{\prime}, \\
\alpha_{2} & =\frac{-1}{\pi} u *\left[\frac{2 x y}{\left(x^{2}+y^{2}\right)^{2}}\right], \beta_{2}=\frac{-1}{\pi} u *\left[\frac{x^{2}-y^{2}}{\left(x^{2}+y^{2}\right)^{2}}\right],
\end{aligned}
$$

here $*$ is the convolution operator. It can be shown that $\mathcal{T}(u)$ lies in $\mathcal{B}^{\alpha}(\widehat{\mathbb{D}})$ (see [7]) and $\mathrm{H} 2 \mathrm{a}$ is satisfied. The above properties are also easily checked for the first example (rigid top instead of free surface on the upper layer).

\subsubsection{Resolvent operator for large $\varepsilon|k|$}

We finally give the behavior of the resolvent operator on the imaginary axis for $|k| \geq$ $\delta / 2 \varepsilon$.

Hypothesis $\mathbf{H 3}$ (resolvent operator for large $\varepsilon|k|$ ) Let $V \in \mathbb{H}$, then, for $k$ real

$$
\left(i k-L_{\varepsilon}\right)^{-1} V=G(\varepsilon, k)(V),
$$


where $k \mapsto G(\varepsilon, k)$ is continuously differentiable in $\mathcal{L}(\mathbb{H}, \mathbb{D})$ for $\varepsilon|k| \geq \delta / 2$ with the following estimates in $\mathcal{L}(\mathbb{H})$, and in $\mathcal{L}(\widetilde{\mathbb{H}}, \widehat{\mathbb{D}})$

$$
\begin{aligned}
\left\|\frac{\partial^{n} G}{\partial k^{n}}\right\|_{\mathcal{L}(\mathbb{H})} & \leq \frac{c}{|k|^{n+1}}, n=0,1,2,3, \\
\left\|\frac{\partial^{n} G}{\partial k^{n}}\right\|_{\mathcal{L}(\widetilde{\mathbb{H}}, \widehat{\mathbb{D}})} & \leq \frac{c \varepsilon}{|k|^{n}}, n=0,1,2,3 .
\end{aligned}
$$

The above assumption means that the resolvent operator behaves like $1 / k$ in $\mathcal{L}(\mathbb{H})$ and that we obtain a bound of the norm from $\widetilde{\mathbb{H}}$ into $\widehat{\mathbb{D}}$ in multiplying by $\varepsilon k$. Hypothesis H3 is satisfied for our examples, as can be checked for instance in using a detailed computation of the resolvent as in [5].

\section{Bifurcation Problem}

Let us come back to the study of the reversible dynamical system (3.1) where $\mathcal{L}_{\varepsilon}$ satisfies the hypothesis $\mathrm{H} 1, \mathrm{H} 2, \mathrm{H} 3$ of section 3 . In section 4.1 we give precisions on the nonlinear term $\mathcal{N}_{\varepsilon}$. In section 4.2 we decompose the solutions as

$$
U=(\nu+w) \xi_{0}+\varepsilon Y,
$$

and find, for a fixed scalar $\nu$, an equivalent system for $w$ and $Y$. We show below that for the scalar $\nu$ close enough to 0 , and for solutions tending towards 0 at infinity, then $w$ is a solution of a perturbation of the Benjamin-Ono equation and $Y$ is a function of $w$.

\subsection{Hypothesis on the nonlinear term}

Consider the differential equation (3.1) in the Banach space $\mathbb{H}$, then we make the following assumption on the nonlinear term $\mathcal{N}_{\varepsilon}$, divided in two parts.

Hypothesis H4 Required properties for the nonlinear operator $\mathcal{N}_{\varepsilon}$ Hypothesis H4a (regularity of $\mathcal{N}_{\varepsilon}$ ): For $k \geq 3$, the nonlinear map $\mathcal{N}_{\varepsilon}$ satisfies

$$
\begin{aligned}
\mathcal{N}_{\varepsilon} & \in \mathcal{C}^{k}\left(\mathcal{B}^{\alpha}(\widehat{\mathbb{D}}), B_{3}^{\alpha}(\widetilde{\mathbb{H}})\right), \quad D_{U} \mathcal{N}_{\varepsilon}(0)=0, \\
D_{U}^{m} \mathcal{N}_{\varepsilon}(0) & =O\left(\varepsilon^{m-2}\right), \quad m=2,3 .
\end{aligned}
$$

In particular, we have

$$
\mathcal{N}_{\varepsilon}(U)=N_{2}(U, U)+O(\varepsilon) \text { for }\|U\|_{\mathcal{B}} \leq M,
$$

where $\mathrm{N}_{2}$ is quadratic.

Remark 4a. It follows from H4a, in fixing $x$, that $\mathcal{N}_{\varepsilon} \in \mathcal{C}^{k}(\widehat{\mathbb{D}}, \widetilde{\mathbb{H}})$. Notice that the above estimates on the derivative of the nonlinear term result from the original scaling $\underline{U}=\varepsilon U$ made before obtaining the form (3.1) of the system. Assumption H4a also sets that $\mathcal{N}_{\varepsilon}(U)$ decays at least as $1 /|x|^{3}$ as $|x| \rightarrow \infty$ when $U$ in $\mathcal{B}^{\alpha}(\widehat{\mathbb{D}})$ roughly decays as $1 /|x|^{2}$. This is quite natural since the nonlinear terms are at least quadratic. Finally hypothesis H4a allows to show the following 
Lemma 4.1 (family of stationary reversible solutions). Assume that $\mathcal{L}_{\varepsilon}$ satisfies hypothesis $H_{1}$ and that $\mathcal{N}_{\varepsilon}$ satisfies hypothesis $\mathrm{H}_{4}$ a. Then there exists near the origin a smooth one-parameter family of stationary reversible solutions in $\mathbb{D}$ of (3.1). Moreover, there exists a smooth change of coordinates in $\mathbb{D}$ such that in the new coordinates, this family becomes

$$
U=\nu \xi_{0}
$$

$\nu \in \mathbb{R}$ being in a neighborhood of 0 .

Proof. The proof is an adaptation of the Lyapunov-Schmidt method with the following ingredients:

i) the decomposition of $\mathbb{D}$ on $\operatorname{ker} p_{0}^{*} \oplus\left\{\xi_{0}\right\}$,

ii) the property that when $U$ satisfies $S U=U$, then $\mathcal{N}_{\varepsilon}(U)$ lies in the range of $\mathcal{L}_{\varepsilon}:$

$$
\mathcal{N}_{\varepsilon}(U) \in \widetilde{\mathbb{H}}, \quad \xi_{\varepsilon}^{*}\left(\mathcal{N}_{\varepsilon}(U)\right)=0, \quad \eta_{\varepsilon}^{*}\left(\mathcal{N}_{\varepsilon}(U)\right)=0,
$$

(see the remark below lemma 3.1). We can then show that there exists $\nu_{0}>0$, such that for $|\nu|<\nu_{0}$ there is a family of symmetric equilibria of the form

$$
U=\nu \xi_{0}+\mathcal{Y}_{\nu}, \quad \mathcal{Y}_{\nu} \in \operatorname{ker} p_{0}^{*} \cap \mathbb{D}, \quad \mathcal{Y}_{\nu}=O\left(\nu^{2}\right) .
$$

Now, the change of variables

$$
\left\{\begin{array}{l}
U=V+\mathcal{Y}_{p_{0}^{*}(V)}, \\
V=U-\mathcal{Y}_{p_{0}^{*}(U)},
\end{array}\right.
$$

shows that $V=\nu \xi_{0}$ is a solution of the new reversible system

$$
\begin{aligned}
\frac{d V}{d x}= & \left(\mathbb{I}-\frac{d \mathcal{Y}_{p_{0}^{*}(V)}}{d \nu} p_{0}^{*}\right)\left\{\mathcal{L}_{\varepsilon} V+\mathcal{N}_{\varepsilon}\left(V+\mathcal{Y}_{p_{0}^{*}(V)}\right)\right\}+ \\
& \left.-\mathcal{N}_{\varepsilon}\left(p_{0}^{*}(V) \xi_{0}+\mathcal{Y}_{p_{0}^{*}(V)}\right)\right\} .
\end{aligned}
$$

We observe that the linear operator on the right-hand side of this equation is still $\mathcal{L}_{\varepsilon}$. Let us denote the new nonlinear term by $\widetilde{\mathcal{N}}_{\varepsilon}$. We then check that the estimates of hypothesis H4a are not satisfied by $\widetilde{\mathcal{N}}_{\varepsilon}$ : indeed we have $D_{U}^{3} \widetilde{\mathcal{N}}_{\varepsilon}(0)=O(1)$ instead of $O(\varepsilon)$. For having H4a totally satisfied by $\widetilde{\mathcal{N}_{\varepsilon}}$, it is sufficient to assume $N_{2}\left(\xi_{0}, \xi_{0}\right)=0$. In the following, instead of making this (quite small) assumption, we make the equivalent

Hypothesis H4b (simplification hypothesis) The nonlinear operator $\mathcal{N}_{\varepsilon}$ satisfies $\mathcal{N}_{\varepsilon}\left(\nu \xi_{0}\right)=0$ for all $\nu \in \mathbb{R}$ in a neighborhood of 0 .

Remark 4b. This assumption H4b corresponds to the existence of a family of stationary reversible solutions of (3.1) : $U=\nu \xi_{0}, \nu \in \mathbb{R}$. This assumption is not a strong one, as we noticed above.

We now need to connect the nonlinear operator $\mathcal{N}_{\varepsilon}$ and the vector $\theta_{k}$ occurring in hypothesis H1c and H2a:

Hypothesis $\mathbf{H} 5$ (link between $\mathcal{N}_{\varepsilon}$ and $\boldsymbol{\theta}_{k}$ ). Defining the linear operators $D_{\varepsilon}$ and $T_{\varepsilon}^{\nu} \in \mathcal{L}(\widehat{\mathbb{D}}, \widetilde{\mathbb{H}})$ and the vector function $\tau_{\varepsilon}^{\nu}(k)$ by

$$
\begin{aligned}
D_{U} \mathcal{N}_{\varepsilon}\left(\nu \xi_{0}\right) & =T_{\varepsilon}^{\nu}, \\
D_{U}^{2} \mathcal{N}_{\varepsilon}(0)\left(\xi_{0}, \cdot\right) & =D_{\varepsilon}, \\
D_{U} \mathcal{N}_{\varepsilon}\left(\nu \xi_{0}\right) \theta_{k} & =\nu \tau_{\varepsilon}^{\nu}(k),
\end{aligned}
$$


then we assume for $\varepsilon|k|<\delta$

$$
\begin{aligned}
k & \mapsto \tau_{\varepsilon}^{\nu}(k) \in \mathcal{C}_{\lim }^{3}(\mathbb{R}, \widetilde{\mathbb{H}}) \text { for }|\nu|<\nu_{0}, \\
\xi_{\varepsilon}^{*}\left(D_{\varepsilon} \theta_{k}\right) & =2 c_{0}+\varepsilon \gamma_{\varepsilon}(k), \quad c_{0} \neq 0, \quad k \mapsto \gamma_{\varepsilon}(k) \in \mathcal{C}_{\lim , \varepsilon}^{2}(\mathbb{R}, \mathbb{R}) .
\end{aligned}
$$

Examples: In the two examples mentioned above, the hypothesis $\mathrm{H} 4 \mathrm{~b}$ is satisfied. The family of equilibria $\nu \xi_{0}$ for $\nu \in \mathbb{R}$, corresponds to uniform flows of the upper layer moving horizontally over the bottom layer at rest.

For checking hypothesis $\mathrm{H} 5$, let us only consider the example detailed at section 2. We obtain the following for $\tau_{\varepsilon}^{\nu}(k), D_{\varepsilon}, T_{\varepsilon}^{\nu}$, and $c_{0}$, where it is clear that H4a and H5 are satisfied:

$$
\begin{aligned}
D_{\varepsilon} Y= & \left(\left.3 \rho \frac{\partial \alpha_{1}}{\partial y}\right|_{y=0}, 2 \alpha_{11},-\frac{2 \lambda}{b} \widetilde{Z},-\frac{\partial \beta_{1}}{\partial y}, \frac{\partial \alpha_{1}}{\partial y}, 0,0\right)^{t}, \\
T_{\varepsilon}^{\nu} Y= & \varepsilon^{-1}\left(-\left.\rho \frac{\partial \alpha_{1}}{\partial y}\right|_{y=0}\left(e^{-3 \varepsilon \nu}-1\right), \alpha_{11}\left(e^{2 \varepsilon \nu}-1\right), \frac{\lambda}{b} \widetilde{Z}\left(e^{-2 \varepsilon \nu}-1\right),\right. \\
& \left.\frac{\partial \beta_{1}}{\partial y}\left(e^{-\varepsilon \nu}-1\right),-\frac{\partial \alpha_{1}}{\partial y}\left(e^{-\varepsilon \nu}-1\right), 0,0\right), \\
\xi_{\varepsilon}^{*}\left(D_{\varepsilon} \theta_{k}\right)= & -\frac{\lambda}{\lambda-1}(3-\varepsilon)=-\frac{1}{a}(3-\varepsilon), \quad c_{0}=-3 a^{-1}, \\
\tau_{\varepsilon}^{\nu}(k)= & \varepsilon^{-1}\left(\frac{\rho \lambda}{\lambda-1}\left(e^{-3 \varepsilon \nu}-1\right), \frac{-1}{\lambda-1}\left(e^{2 \varepsilon \nu}-1\right), 0,0, \frac{\lambda}{\lambda-1}\left(e^{-\varepsilon \nu}-1\right), 0,0\right) .
\end{aligned}
$$

The fact that $\xi_{\varepsilon}^{*}\left(D_{\varepsilon} \theta_{k}\right)$ and $\tau_{\varepsilon}^{\nu}(k)$ are independent of $k$ in the water wave examples comes from the existence of the Bernoulli first integral (see (2.5)). Indeed it can be seen in section 4 that the coefficient $\xi_{\varepsilon}^{*}\left(D_{\varepsilon} \theta_{k}\right)$ appears in (4.32) which results from the differentiation with respect to $x$ of the Bernoulli first integral and then gives a local quadratic term for the equation for $w$.

In the following, we search for solutions of (3.1) of the form

$$
U=(\nu+w) \xi_{0}+\varepsilon Y, p_{0}^{*}(Y)=0 .
$$

Thanks to hypothesis H4, the nonlinear term computed for a vector $U$ given by (4.1), leads to a smooth operator $R_{\varepsilon}^{\nu}$ defined by

$$
\mathcal{N}_{\varepsilon}\left((\nu+w) \xi_{0}+\varepsilon Y\right)=\varepsilon T_{\varepsilon}^{\nu}(Y)+\varepsilon R_{\varepsilon}^{\nu}(w, Y),
$$

with the following properties

Proposition 4.2 (properties of $\left.R_{\varepsilon}^{\nu}\right)$. The map $R_{\varepsilon}^{\nu}: B_{2}^{\alpha}(\mathbb{R}) \times \mathcal{B}^{\alpha}(\widehat{\mathbb{D}}) \rightarrow B_{3}^{\alpha}(\widetilde{\mathbb{H}})$ is $\mathcal{C}^{k}$ and satisfies

$$
R_{\varepsilon}^{\nu}(w, Y)=w D_{\varepsilon} Y+\widetilde{R}_{\varepsilon}^{\nu}(w, Y),
$$

with

$$
(u, Y) \mapsto u D_{\varepsilon} Y \in \mathcal{L}\left(B_{2}^{\alpha}(\mathbb{R}) \times \mathcal{B}^{\alpha}(\widehat{\mathbb{D}}), B_{3}^{\alpha}(\widetilde{\mathbb{H}})\right),
$$

and

$$
\left\|\widetilde{R}_{\varepsilon}^{\nu}(w, Y)\right\|_{3, \widetilde{\mathbb{H}}}^{\alpha} \leq c \varepsilon\|Y\|_{\mathcal{B}}\left(\|w\|_{2, \mathbb{R}}^{1, \alpha}+\|Y\|_{\mathcal{B}}\right),
$$

for $\|w\|_{2, \mathbb{R}}^{1, \alpha}+\|Y\|_{\mathcal{B}} \leq M$ and $|\nu|<\nu_{0}$. 
We notice that the main term of $R_{\varepsilon}^{\nu}$ is $u D_{\varepsilon} Y$. Indeed there is no quadratic term in $w$ because of $\mathrm{H} 4 \mathrm{~b}$. The linear map $D_{\varepsilon}$ introduced in hypothesis $\mathrm{H} 5$ and proposition 4.2 will play an important role in the sequel.

\subsection{Main Theorem}

We search solutions written as (4.1) where $\nu$ is a parameter close to 0 . Thanks to the definition of $R_{\varepsilon}^{\nu}$, equation (3.1) reads

$$
\begin{aligned}
& \frac{d w}{d x}=p_{0}^{*}\left(\varepsilon \mathcal{L}_{\varepsilon} Y\right)+p_{0}^{*}\left(T_{\varepsilon}^{\nu} Y\right)+\varepsilon p_{0}^{*}\left(R_{\varepsilon}^{\nu}(w, Y)\right), \\
& \frac{d Y}{d x}=\pi \mathcal{L}_{\varepsilon} Y+\pi T_{\varepsilon}^{\nu} Y+\pi R_{\varepsilon}^{\nu}(w, Y),
\end{aligned}
$$

where we use the projection $\pi=\mathbb{I}-\xi_{0} p_{0}^{*}$.

From now on we are only interested in reversible solutions $(w, Y)$ of (4.3)-(4.4) which tend towards 0 as $|x| \rightarrow \infty$. The reversibility of a solution $U$ means that

$$
w \text { is even and } S Y(x)=Y(-x) .
$$

We expect $w$ and $Y$ to tend towards 0 as $1 / x^{2}$, which explains our choice of spaces. We now state the main result of this section.

Theorem 4.3. Assume that $\mathcal{L}_{\varepsilon}$ satisfies hypotheses $H 1, H 2$ and $H 3$ and that $\mathcal{N}_{\varepsilon}$ satisfies hypothesis $H_{4}, \mathrm{H} 5$. Then, for any $0<\alpha<1$, there exists $\varepsilon_{0}>0$ and $\nu_{0}>0$ such that, for $0<\varepsilon<\varepsilon_{0}$ and for $0 \leq|\nu|<\nu_{0} \varepsilon$, equation (3.1) has a weak reversible solution $U_{\varepsilon, \nu}$ which satisfies

$$
U_{\varepsilon, \nu}(x)=\left(\nu+u_{h}(x)\right) \xi_{0}+\widetilde{U}_{\varepsilon, \nu}(x),
$$

where $u_{h}$ is the Benjamin-Ono homoclinic given by (1.3), $\xi_{0}$ is the (symmetric) eigenvector associated with the 0 eigenvalue, and $\widetilde{U}_{\varepsilon, \nu} \in B_{2}^{1, \alpha}(\mathbb{R}) \xi_{0} \oplus \mathcal{B}^{\alpha}(\widehat{\mathbb{D}})$ (hence homoclinic to 0) is $O\left(\varepsilon+|\nu| \varepsilon^{-1}\right)$. Moreover, if $\tau_{\varepsilon}^{u}(k)$ is independent of $k$, then $\widetilde{U}_{\varepsilon, \nu}=O(\varepsilon+|\nu|)$.

A solution in $B_{2}^{1, \alpha}(\mathbb{R}) \xi_{0} \oplus \mathcal{B}^{\alpha}(\widehat{\mathbb{D}})$ means that $(4.4)$ is only satisfied in a weak sense, since $\mathcal{L}_{\varepsilon} Y(x)$ is not defined in $\mathbb{H}$ while $R_{\varepsilon}^{\nu}(w, Y)$ and $T_{\varepsilon}^{\nu} Y$ are well defined. Indeed, for $k$ real $\neq 0, \widehat{Y}(k)$ lies in $\mathbb{D}$, while we can only say that $Y(x) \in \widehat{\mathbb{D}}$. In the water wave examples, we recover the equation (4.4) valid in $\mathbb{H}$ because of the regularity properties of harmonic functions in the half-plane, which means that our solutions $U_{\varepsilon, \nu}$ are continuous functions of $x$ taking values in $\mathbb{D}$.

Example. This theorem applies in our two water wave examples. In the explicit example of section 2, for $\varepsilon>0$ small enough, there exists a reversible solution in $B_{2}^{1, \alpha}(\mathbb{R}) \xi_{0} \oplus \mathcal{B}^{\alpha}(\widehat{\mathbb{D}})$ of $(2.4)$ of the form

$$
U_{\varepsilon, \nu}=\left(\nu+u_{h 1}\right) \xi_{0}+\widetilde{U}_{\varepsilon, \nu}, \quad \widetilde{U}_{\varepsilon, \nu}=O(\varepsilon+|\nu|),
$$

with

$$
u_{h 1}=\frac{4 \tilde{\rho}^{2}}{3\left(\tilde{\rho}^{2}+x^{2}\right)},
$$


and

$$
\tilde{\rho}=\frac{\lambda-1}{\lambda}=\frac{\rho-\varepsilon}{1-\varepsilon} .
$$

The expression of $Z=(1 / 2 \lambda)\left(1-e^{-2 \lambda \widetilde{Z}}\right)$ together with $\widetilde{Z}(\underline{x}) \sim \frac{\varepsilon}{\lambda}\left\{\nu+u_{h 1}(\varepsilon \underline{x})\right\}$ gives, for the free surface, in unscaled variables

$$
Z(\underline{x}) \sim \frac{\varepsilon}{\lambda}\left(\nu+\frac{4 \tilde{\rho}^{2}}{3\left(\tilde{\rho}^{2}+\varepsilon^{2} \underline{x}^{2}\right)}\right),
$$

The expression of the interface satisfies $\frac{d Z_{I}}{d x} \sim \alpha_{10}$, then

$$
Z_{I}(\underline{x}) \sim-\frac{(\lambda-1) \varepsilon}{\lambda}\left\{\nu+\frac{4 \tilde{\rho}^{2}}{3\left(\tilde{\rho}^{2}+\varepsilon^{2} \underline{x}^{2}\right)}\right\} .
$$

Note that these formula fit with the imposed flux $(=1)$ at infinity. Also note that this result is different from the one of [7] since the reversible solution tends towards an equilibrium at infinity in our case, contrary to the fact that in [7] the limit at infinity is a periodic wave.

\subsection{Proof of Theorem 4.3}

The aim of this section is to prove Theorem 4.3. This is done in four steps. First we study the linear system. Then we reduce (3.1) to an equation for $w$ in showing that $Y$ can be written as a function of $w, \nu$ and $\varepsilon$. In section 4.3 .3 we prove that the equation for $w$ is a perturbation of the Benjamin-Ono equation. We finally solve this new equation for $w$ by the implicit function Theorem.

\subsubsection{Linear Lemma}

The previous section leads to the study of the following affine system

$$
\begin{aligned}
\frac{d w}{d x} & =p_{0}^{*}\left(\varepsilon \mathcal{L}_{\varepsilon} Y\right)+p_{0}^{*}\left(T_{\varepsilon}^{\nu} Y\right)+\varepsilon p_{0}^{*}(R), \\
\frac{d Y}{d x} & =\pi \mathcal{L}_{\varepsilon} Y+\pi T_{\varepsilon}^{\nu} Y+\pi R,
\end{aligned}
$$

with $S R(x)=-R(-x)$ and where we now assume that $R \in B_{3}^{\alpha}(\widetilde{\mathbb{H}})$. Regarding this system we have the following result

Lemma 4.4. Let $R \in B_{3}^{\alpha}(\widetilde{\mathbb{H}})$ such that $S R(x)=-R(-x)$. Let $\mathcal{L}_{\varepsilon}(\varepsilon>0)$ satisfy hypotheses H1, H2 and H3, and $\mathcal{N}_{\varepsilon}$ satisfy hypotheses $H_{4}$ and $H_{5}$. Then a weak (in the sense that it is strong for the Fourier transforms) solution $(w, Y)$ of system (4.5)-(4.6) in $B_{2}^{1, \alpha}(\mathbb{R}) \times \mathcal{B}^{\alpha}(\widehat{\mathbb{D}})$ satisfies

$$
\begin{aligned}
Y & =a \mathcal{T}(w)+\mathcal{T}_{\varepsilon, \nu}(R), \\
w+a \mathcal{H}\left(w^{\prime}\right) & =\int_{-\infty}^{x} \xi_{\varepsilon}^{*}(R(s)) d s+\mathcal{C}_{\varepsilon, \nu}(R),
\end{aligned}
$$


where $\mathcal{T}(w)$ is defined by hypothesis H2, and where the following estimates hold

$$
\begin{aligned}
& \left\|\mathcal{T}_{\varepsilon, \nu}(R)\right\|_{\mathcal{B}} \leq c(\varepsilon+|\nu||\ln \varepsilon|)\|R\|_{3, \widetilde{\mathbb{H}}}^{\alpha}, \\
& \left\|\mathcal{C}_{\varepsilon, \nu}(R)\right\|_{2, \mathbb{R}}^{\alpha} \leq c\left(\varepsilon+|\nu| \varepsilon^{-1}\right)\|R\|_{3, \widetilde{\mathbb{H}}}^{\alpha} \cdot
\end{aligned}
$$

Moreover, if $\tau_{\varepsilon}^{\nu}(k)$ is independent of $k$, then $\mathcal{T}_{\varepsilon, \nu}(R)$ and $\mathcal{C}_{\varepsilon, \nu}(R)$ are $O\left((\varepsilon+|\nu|)\|R\|_{3, \widetilde{\mathbb{H}}}^{\alpha}\right)$.

The system (4.7)-(4.8) is a weaker formulation of system (4.5)-(4.6) since $\mathcal{L}_{\varepsilon} Y$ is not defined for $Y \in \mathcal{B}^{\alpha}(\widehat{\mathbb{D}})$ (while $(4.5)$ is well-defined for $Y \in \mathcal{B}^{\alpha}(\widehat{\mathbb{D}})$ because of the hypothesis $\mathrm{H} 2 \mathrm{~b}$ on $p_{0}^{*}$ ). We denote by "weak" our solutions, since only the Fourier transform of the system is satisfied. We can see in water wave examples that equation (4.6) can be understood in the distributions sense, and that a solution $Y \in \mathcal{B}^{\alpha}(\widehat{\mathbb{D}})$ of (4.6) leads to $x \mapsto Y(x) \in \mathcal{C}^{0}(\mathbb{R}, \mathbb{D}) \cap \mathcal{C}^{1}(\mathbb{R}, \mathbb{H})$.

The result of lemma 4.4 can be understood as the inversion for $\varepsilon>0$ of the linear operator

$$
U \mapsto \frac{d U}{d x}-\mathcal{L}_{\varepsilon} U
$$

in a suitable space (since equation (4.8) can be solved with respect to $w$ by using the Fourier transform). Coming back to the original linearized operator before the scaling, for allowing $\varepsilon$ to be negative, the linear operator $\frac{d U}{d x}-L_{\varepsilon} U$ is invertible for $\varepsilon>0$, and not invertible for $\varepsilon<0$, because of the imaginary eigenvalues of $L_{\varepsilon}$. This can be also interpreted in saying that $\varepsilon=0$ is the border of a continuous set of $\varepsilon^{\prime} s$ in which the above operator is not invertible, analogously to the type of work developed by Stuart [11].

To prove lemma 4.4 we use the Fourier transform of (4.5)-(4.6), and consider separately the cases $\varepsilon|k| \leq \delta$ and $\varepsilon|k| \geq \delta$, in order to use the hypothesis made on the resolvent operator.

Proof of lemma 4.4. Taking the Fourier transform, we obtain from (4.5)-(4.6)

$$
\left(i k-\left(\mathcal{L}_{\varepsilon}+T_{\varepsilon}^{\nu}\right)\right)\left(\widehat{w} \xi_{0}+\varepsilon \widehat{Y}\right)=\varepsilon \widehat{R} .
$$

We now introduce a splitting of unity, with regular even functions $\varphi_{0}$ and $\varphi_{1}$ such that

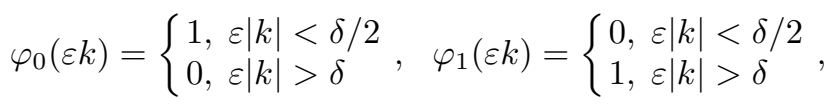

and $\varphi_{0}+\varphi_{1}=1$.

Step $1: \varepsilon|k| \leq \delta$. We first consider the equation

$$
\left(i k-\left(\mathcal{L}_{\varepsilon}+T_{\varepsilon}^{\nu}\right)\right)\left(\widehat{w}_{0} \xi_{0}+\varepsilon \widehat{Y}_{0}\right)=\varepsilon \varphi_{0}(k \varepsilon) \widehat{R},
$$

where we defined $\widehat{w}_{0}=\varphi_{0}(k \varepsilon) \widehat{w}$ and $\widehat{Y}_{0}=\varphi_{0}(k \varepsilon) \widehat{Y}$. Here we see that we need the resolvent $\left(i k-\left(\mathcal{L}_{\varepsilon}+T_{\varepsilon}^{\nu}\right)\right)^{-1}$ of the linear operator $\mathcal{L}_{\varepsilon}+T_{\varepsilon}^{\nu}$ for $k$ real and $0<\varepsilon|k|<\delta$. We use the Hypothesis H1 to compute $\left(i k-\left(\mathcal{L}_{\varepsilon}+T_{\varepsilon}^{\nu}\right)\right)^{-1}$ for $|\nu|$ small enough and $0<\varepsilon|k|<\delta$, as a perturbation of $\left(i k-\mathcal{L}_{\varepsilon}\right)^{-1}$. 
Proposition 4.5 (Resolvent of $\mathcal{L}_{\varepsilon}+T_{\varepsilon}^{\nu}$ for $0<\varepsilon|k|<\delta$ ). Let $V \in \mathbb{H}$, $k$ real such that $0<\varepsilon|k|<\delta$ and $|\nu|<\nu_{0}$, then

$$
\begin{aligned}
\left(i k-\left(\mathcal{L}_{\varepsilon}+T_{\varepsilon}^{\nu}\right)\right)^{-1} V= & \frac{\xi_{\varepsilon, k}^{*}(V)}{i k \varepsilon \Delta} \xi_{0}+\frac{\eta_{\varepsilon, k}^{*}(V)}{\Delta} \theta_{k}+\varepsilon S_{\varepsilon, k}(V) \\
& +\frac{\xi_{\varepsilon, k}^{*}\left(\Gamma_{\varepsilon, k}^{\nu}(V)\right)}{i k \varepsilon \Delta} \xi_{0}+\frac{\eta_{\varepsilon, k}^{*}\left(\Gamma_{\varepsilon, k}^{\nu}(V)\right)}{\Delta} \theta_{k}+\varepsilon S_{\varepsilon, k}\left(\Gamma_{\varepsilon, k}^{\nu}(V)\right),
\end{aligned}
$$

where

$$
\Gamma_{\varepsilon, k}^{\nu}(V)=\frac{\nu}{\Delta} H_{\varepsilon, k}^{\nu}(V)+\varepsilon \nu I_{\varepsilon, k}^{\nu}(V),
$$

where $k \mapsto H_{\varepsilon, k}^{\nu}$ and $k \mapsto I_{\varepsilon, k}^{\nu}$ are in $\mathcal{C}^{0}(\mathbb{R} \backslash\{0\}, \mathcal{L}(\mathbb{H})) \cap \mathcal{C}_{\text {lim }}^{3}(\mathbb{R}, \mathcal{L}(\widetilde{\mathbb{H}}))$ with the following estimates for $n=0,1,2,3$

$$
\begin{aligned}
& \sup _{0<\varepsilon|k|<\delta}\left\|H_{\varepsilon, k}^{\nu}{ }^{(n)}\right\|_{\widetilde{\mathbb{H}}} \leq c, \\
& \sup _{0<\varepsilon|k|<\delta}\left\|I_{\varepsilon, k}^{\nu}{ }^{(n)}\right\|_{\widetilde{\mathbb{H}}} \leq c\left(|\nu|+(\varepsilon+|\nu|)^{n}\right) .
\end{aligned}
$$

Moreover the following symmetry properties hold

$$
\begin{aligned}
S H_{\varepsilon, k}(V) & =H_{\varepsilon,-k}(S V), \\
S I_{\varepsilon, k}(V) & =I_{\varepsilon,-k}(S V) .
\end{aligned}
$$

Proof. This is straightforward, in using the properties of the form $\eta_{\varepsilon, k}^{*}$ (see H1c), of the vector $\tau_{\varepsilon}^{\nu}(k)$ (see H5), and of the operators $S_{\varepsilon, k}$ (see H1d) and $T_{\varepsilon}^{\nu}$ (see H5). In particular we can see that for $U=w \xi_{0}+Y, p_{0}^{*}(Y)=0$, the equation

$$
\left(i k-\mathcal{L}_{\varepsilon}\right) U=V+T_{\varepsilon}^{\nu} U
$$

leads to the property (notice that $T_{\varepsilon}^{\nu} \xi_{0}=0$ )

$$
T_{\varepsilon}^{\nu} Y=\Gamma_{\varepsilon, k}^{\nu}(V) .
$$

We deduce from proposition (4.5) that

$$
\widehat{w}_{0}(k)=\frac{\xi_{\varepsilon}^{*}(\widehat{R})}{i k \Delta} \varphi_{0}+S_{w}^{(0)}(\widehat{R})+S_{w, \nu}^{(0)}(\widehat{R}),
$$

where

$$
S_{w}^{(0)}(\widehat{R})=-\varphi_{0} \frac{i \varepsilon \operatorname{sgn}(k)}{\Delta} \chi_{\varepsilon}^{*}(\widehat{R})+\varphi_{0} \frac{\zeta_{\varepsilon, k}^{*}(\widehat{R})}{i k \Delta},
$$

and

$$
S_{w, \nu}^{(0)}(\widehat{R})=\frac{\xi_{\varepsilon, k}^{*}\left(\Gamma_{\varepsilon, k}^{\nu}(\widehat{R})\right)}{i k \Delta} \varphi_{0} .
$$

Similarly, in using (3.6), we obtain the following equation for $\widehat{Y}_{0}$

$$
\widehat{Y}_{0}(k)=-a \frac{\xi_{\varepsilon}^{*}(\widehat{R})}{\Delta} \varphi_{0} \theta_{k}+S_{Y}^{(0)}(\widehat{R})+S_{Y, \nu}^{(0)}(\widehat{R}),
$$


where

$$
S_{Y}^{(0)}(\widehat{R})=\frac{\varepsilon \chi_{\varepsilon}^{*}(\widehat{R})}{\Delta} \varphi_{0} \theta_{k}+\varphi_{0} \frac{\beta_{\varepsilon, k}^{*}(\widehat{R})}{\Delta} \theta_{k}+\varepsilon \varphi_{0} S_{\varepsilon, k}(\widehat{R}),
$$

and

$$
S_{Y, \nu}^{(0)}(\widehat{R})=\frac{\eta_{\varepsilon, k}^{*}\left(\Gamma_{\varepsilon, k}^{\nu}(\widehat{R})\right)}{\Delta} \varphi_{0} \theta_{k}+\varepsilon \varphi_{0} S_{\varepsilon, k}\left(\Gamma_{\varepsilon, k}^{\nu}(\widehat{R})\right) .
$$

These terms are controlled via the following lemma

Lemma 4.6. With the assumptions of lemma 4.4, and for $0<\varepsilon|k|<\delta$, and $|\nu|<\nu_{0}$, we have

$$
\begin{aligned}
& \mathcal{F}^{-1}\left(S_{w}^{(0)}(\widehat{R})\right), \mathcal{F}^{-1}\left(S_{w, u}^{(0)}(\widehat{R})\right) \in B_{2}^{1, \alpha}(\mathbb{R}), \\
& \mathcal{F}^{-1}\left(S_{Y}^{(0)}(\widehat{R})\right), \mathcal{F}^{-1}\left(S_{Y, u}^{(0)}(\widehat{R})\right) \in \mathcal{B}^{\alpha}(\widehat{\mathbb{D}}),
\end{aligned}
$$

with the estimates

$$
\begin{aligned}
\left\|\mathcal{F}^{-1}\left(S_{w}^{(0)}(\widehat{R})\right)\right\|_{2, \mathbb{R}}^{1, \alpha} & \leq c \varepsilon\|R\|_{3, \widetilde{H}}^{\alpha}, \\
\left\|\mathcal{F}^{-1}\left(S_{w, \nu}^{(0)}(\widehat{R})\right)\right\|_{2, \mathbb{R}}^{1, \alpha} & \leq c(\varepsilon+|\nu \| \ln \varepsilon|)\|R\|_{3, \widetilde{\mathbb{H}}}^{\alpha}, \\
\left\|\mathcal{F}^{-1}\left(S_{Y}^{(0)}(\widehat{R})\right)\right\|_{\mathcal{B}} & \leq c \varepsilon\|R\|_{3, \widetilde{\mathbb{H}}}^{\alpha}, \\
\left\|\mathcal{F}^{-1}\left(S_{Y, \nu}^{(0)}(\widehat{R})\right)\right\|_{\mathcal{B}} & \leq c(\varepsilon+|\nu \| \ln \varepsilon|)\|R\|_{3, \widetilde{\mathbb{H}}}^{\alpha} .
\end{aligned}
$$

In the case when $\tau_{\varepsilon}^{\nu}(k)$ is independent of $k$, we replace $|\nu||\ln \varepsilon|$ by $|\nu|$ in the estimates.

Proof. We apply Lemma A.2 of Appendix A to $F_{\varepsilon}(k)=\frac{\zeta_{\varepsilon, k}^{*}}{i k} \in \widetilde{\mathbb{H}}^{*}, F_{\varepsilon}(k)=$ $\frac{\beta_{\varepsilon, k}^{*}}{i k} \in \widetilde{\mathbb{H}}^{*}, p=1, F_{\varepsilon}(k)=\varepsilon S_{\varepsilon, k} \in \mathcal{L}(\widehat{\mathbb{D}}, \widetilde{\mathbb{H}}), p=0$. Let us now consider the first terms appearing in $\mathcal{F}^{-1}\left(S_{w}^{(0)}(\widehat{R})\right)(4.16)$ and in $\mathcal{F}^{-1}\left(S_{Y}^{(0)}(\widehat{R})\right)(4.19)$, i.e.

$$
\mathcal{F}^{-1}\left(-i \varepsilon \frac{\operatorname{sgn}(k)}{\Delta} \varphi_{0} \chi_{\varepsilon}^{*}(\widehat{R})\right)=K_{1} * \mathcal{H}\left(\sigma_{1}^{\prime}\right),
$$

where

$$
\sigma_{1}(x)=\int_{-\infty}^{x} \chi_{\varepsilon}^{*}(R) d s, \quad K_{1}(x)=\mathcal{F}^{-1}\left(\frac{\varepsilon \varphi_{0}(k \varepsilon)}{\Delta(\varepsilon, k)}\right)
$$

and

$$
\mathcal{F}^{-1}\left(\frac{\varepsilon}{\Delta} \varphi_{0} \chi_{\varepsilon}^{*}(\widehat{R}) \theta_{k}\right)=\mathcal{F}^{-1}\left(\hat{a} i k \theta_{k}\right),
$$

where $a$ is defined by

$$
a=\mathcal{F}^{-1}\left(\frac{\varepsilon \varphi_{0}}{\Delta} \frac{\chi_{\varepsilon}^{*}(\widehat{R})}{i k}\right)=K_{1} * \sigma_{1} .
$$

Lemma A.2 with $F_{\varepsilon}=1$ proves that $K_{1}$ satisfies lemma A.1 with constants of order $O(\varepsilon)$. Since $R \in B_{3}^{\alpha}(\widetilde{\mathbb{H}})$ is antireversible and $\chi_{\varepsilon}^{*}$ is invariant under $S$, then $\sigma_{1} \in$ $B_{2}^{1, \alpha}(\mathbb{R})$ is an even primitive of $\chi_{\varepsilon}^{*}(R)$. It results from corollary B2 of [7] that

$$
\mathcal{F}^{-1}\left(-i \operatorname{sgn}(k) \chi_{\varepsilon}^{*}(\widehat{R})\right)=\mathcal{H}\left(\sigma_{1}^{\prime}\right) \in B_{2}^{\alpha}(\mathbb{R}),
$$


with

$$
\left\|\mathcal{F}^{-1}\left(-i \operatorname{sgn}(k) \chi_{\varepsilon}^{*}(\widehat{R})\right)\right\|_{2, \mathbb{R}}^{\alpha} \leq c\left\|\sigma_{1}\right\|_{2, \mathbb{R}}^{1, \alpha} \leq c\|R\|_{3, \widetilde{\mathbb{H}}^{\prime}}^{\alpha}
$$

Finally, lemma A.1 shows

$$
\mathcal{F}^{-1}\left(\frac{\varepsilon}{\Delta} \varphi_{0} \chi_{\varepsilon}^{*}(\widehat{R}) \theta_{k}\right) \in \mathcal{B}^{\alpha}(\widehat{\mathbb{D}}), \quad \mathcal{F}^{-1}\left(-i \varepsilon \frac{\operatorname{sgn}(k)}{\Delta} \varphi_{0} \chi_{\varepsilon}^{*}(\widehat{R})\right) \in B_{2}^{1, \alpha}(\mathbb{R}),
$$

with

$$
\begin{array}{r}
\left\|\mathcal{F}^{-1}\left(-i \varepsilon \frac{\operatorname{sgn}(k)}{\Delta} \varphi_{0} \chi_{\varepsilon}^{*}(\widehat{R})\right)\right\|_{2, \mathbb{R}}^{1, \alpha} \leq c \varepsilon\|R\|_{3, \widetilde{\mathbb{H}}}^{\alpha} \\
\left\|\mathcal{F}^{-1}\left(\frac{\varepsilon}{\Delta} \varphi_{0} \chi_{\varepsilon}^{*}(\widehat{R}) \theta_{k}\right)\right\|_{\mathcal{B}} \leq c \varepsilon\|R\|_{3, \widetilde{\mathbb{H}}}^{\alpha} .
\end{array}
$$

This ends the estimates for $\mathcal{F}^{-1}\left(S_{w}^{(0)}(\widehat{R})\right)$ and $\mathcal{F}^{-1}\left(S_{Y}^{(0)}(\widehat{R})\right)$. For obtaining the estimates on $\mathcal{F}^{-1}\left(S_{w, \nu}^{(0)}(\widehat{R})\right)$ and $\mathcal{F}^{-1}\left(S_{Y, \nu}^{(0)}(\widehat{R})\right)$ in $(4.17)$ and (4.20), we proceed in the same way as above with lemmas A.2 and A.3, using the decomposition (4.11) and estimates (4.12), (4.13). In the case when $\tau_{\varepsilon}^{\nu}(k)$ is independent of $k$, the main terms in $H_{\varepsilon, k}^{\nu}$ and $I_{\varepsilon, k}^{\nu}$ have derivatives of order $O\left(\varepsilon^{n}\right)$ instead of the orders indicated in (4.12), (4.13).

It results from (4.15) and (4.18) that $\widehat{Y}_{0}$ can be written as

$$
\widehat{Y}_{0}=-a i k \theta_{k} \widehat{w}_{0}+\widehat{\mathcal{T}}_{\varepsilon, \nu, 0}(R),
$$

where

$$
\begin{aligned}
\mathcal{T}_{\varepsilon, \nu, 0}(R)= & \mathcal{F}^{-1}\left(a i k S_{w}^{(0)}(\widehat{R}) \theta_{k}\right)+\mathcal{F}^{-1}\left(S_{Y}^{(0)}(\widehat{R})\right) \\
& \left.+\mathcal{F}^{-1}\left(S_{Y, \nu}^{(0)}(\widehat{R})\right)+a \mathcal{F}^{-1}\left(i k S_{w, \nu}^{(0)}(\widehat{R}) \theta_{k}\right)\right)
\end{aligned}
$$

and hypothesis H2a shows that $\mathcal{T}_{\varepsilon, \nu, 0}(R) \in \mathcal{B}^{\alpha}(\widehat{\mathbb{D}})$ with the estimate

$$
\begin{aligned}
\left\|\mathcal{T}_{\varepsilon, \nu, 0}(R)\right\|_{\mathcal{B}} \leq & c\left\|\mathcal{F}^{-1}\left(S_{w}^{(0)}(\widehat{R})\right)\right\|_{2, \mathbb{R}}^{1, \alpha}+\left\|\mathcal{F}^{-1}\left(S_{Y}^{(0)}(\widehat{R})\right)\right\|_{\mathcal{B}} \\
& +\left\|\mathcal{F}^{-1}\left(S_{Y, \nu}^{(0)}(\widehat{R})\right)\right\|_{\mathcal{B}}+c\left\|\mathcal{F}^{-1}\left(S_{w, \nu}^{(0)}(\widehat{R})\right)\right\|_{2, \mathbb{R}}^{1, \alpha}, \\
\leq & c(\varepsilon+|\nu||\ln \varepsilon|)\|R\|_{3, \widetilde{\mathbb{H}}}^{\alpha} \cdot
\end{aligned}
$$

Notice that when $\tau_{\varepsilon}^{\nu}(k)$ is independent of $k$, we replace $|\nu||\ln \varepsilon|$ by $|\nu|$ in the estimate (4.22). The results (4.21) and (4.22) end the first step of the proof.

Step $2: \varepsilon|k| \geq \delta$. We now study the equation

$$
\left(i k-\left(\mathcal{L}_{\varepsilon}+T_{\varepsilon}^{\nu}\right)\right)\left(\widehat{w}_{1} \xi_{0}+\varepsilon \widehat{Y}_{1}\right)=\varepsilon \varphi_{1}(k \varepsilon) \widehat{R},
$$

where $\widehat{w}_{1}=\varphi_{1} \widehat{w}$ and $\widehat{Y}_{1}=\varphi_{1} \widehat{Y}$. Here we must compute the resolvent of $\mathcal{L}_{\varepsilon}+T_{\varepsilon}^{\nu}$ for $k$ real such that $\varepsilon|k|>\delta / 2$. We use hypothesis H3 to compute $\left(i k-\left(\mathcal{L}_{\varepsilon}+T_{\varepsilon}^{\nu}\right)\right)^{-1}$ for $\varepsilon|k|>\delta / 2(k$ real $)$ and $|\nu|$ small enough. 
Proposition 4.7. Let $V \in \mathbb{H}$ and $k$ real such that $\varepsilon|k|>\delta / 2$, then

$$
\left(i k-\left(\mathcal{L}_{\varepsilon}+T_{\varepsilon}^{\nu}\right)\right)^{-1} V=G(\varepsilon, k)(V)+\widetilde{G}_{\nu}(\varepsilon, k)(V),
$$

where $G(\varepsilon, k)$ is defined in hypothesis $H_{4}$, and where $k \mapsto \widetilde{G}_{\nu}(\varepsilon, k)$ is continuously differentiable in $\mathcal{L}(\mathbb{H}, \mathbb{D})$ for $\varepsilon|k|>\delta / 2$ and satisfies for $|\nu|<\nu_{0}$ and $\varepsilon|k|>\delta / 2$

$$
\left\|\frac{\partial^{n} \widetilde{G}_{\nu}}{\partial k^{n}}\right\|_{\mathcal{L}(\widetilde{\mathbb{H}}, \mathbb{H})} \leq \frac{c \varepsilon|\nu|}{|k|^{n+1}}, n=0,1,2,3,
$$

and

$$
\left\|\frac{\partial^{n} \widetilde{G}_{\nu}}{\partial k^{n}}\right\|_{\mathcal{L}(\widetilde{\mathbb{H}}, \widehat{\mathbb{D}})} \leq \frac{c \varepsilon^{2}|\nu|}{|k|^{n}}, n=0,1,2,3 .
$$

Proof. This is straightforward, in using hypothesis H3.

We deduce from Proposition 4.7

$$
\widehat{w}_{1}(k)=\varepsilon \varphi_{1} p_{0}^{*} G(\varepsilon, k)(\widehat{R})+\varepsilon \varphi_{1} p_{0}^{*} \widetilde{G}_{\nu}(\varepsilon, k)(\widehat{R}),
$$

and

$$
\widehat{Y}_{1}(k)=\varphi_{1} \pi G(\varepsilon, k)(\widehat{R})+\varphi_{1} \pi \widetilde{G}_{\nu}(\varepsilon, k)(\widehat{R}) .
$$

These terms are controlled via the following lemma

Lemma 4.8. Let $R \in B_{2}^{\alpha}(\widetilde{\mathbb{H}})$, then $Y_{1} \in \mathcal{B}^{\alpha}(\widehat{\mathbb{D}})$ and $w_{1} \in B_{2}^{1, \alpha}(\mathbb{R})$ with the estimates

$$
\begin{gathered}
\left\|Y_{1}\right\|_{\mathcal{B}} \leq c \varepsilon\|R\|_{2, \widetilde{\mathbb{H}}}^{\alpha}, \\
\left\|w_{1}\right\|_{2, \mathbb{R}}^{1, \alpha} \leq c \varepsilon\|R\|_{2, \widetilde{\mathbb{H}}^{\circ}}^{\alpha}
\end{gathered}
$$

In particular

$$
\left\|\mathcal{F}^{-1}\left(i k \widehat{w}_{1} \theta_{k}\right)\right\|_{\mathcal{B}} \leq c \varepsilon\|R\|_{2, \widetilde{H}^{-}}^{\alpha}
$$

Proof. We use the Corollary of Lemma B.1 of Appendix B, with $F_{\varepsilon}(k)=$ $\pi G(\varepsilon, k)+\pi \widetilde{G}_{\nu}(\varepsilon, k)$ for the estimate of $Y_{1}$, and $F_{\varepsilon}(k)=G(\varepsilon, k)+\widetilde{G}_{\nu}(\varepsilon, k)$ for the estimate of $w_{1}$.

Step 3 : equation for $Y$. We finally collect (4.21), (4.22), (4.29) and obtain the equation

$$
\widehat{Y}(k)=-a i k \widehat{w}(k) \theta_{k}+\widehat{\mathcal{T}}_{\varepsilon, \nu}(R),
$$

where

$$
\mathcal{T}_{\varepsilon, \nu}(R)=\mathcal{T}_{\varepsilon, \nu, 0}(R)+\mathcal{F}^{-1}\left(a i k \widehat{w}_{1}(k) \theta_{k}\right)+Y_{1} .
$$

Equation (4.30) corresponds to (4.7) in Lemma 4.4 and estimates (4.22)-(4.29) give the first estimate of lemma 4.4 . 
Step 4 : equation for $w$. Applying $\xi_{\varepsilon}^{*}$ on (4.30) we obtain

$$
\xi_{\varepsilon}^{*}(Y)=a \mathcal{H}\left(w^{\prime}\right)+\xi_{\varepsilon}^{*}\left(\mathcal{T}_{\varepsilon, \nu}(R)\right),
$$

and from (4.5)-(4.6) we have

$$
\xi_{\varepsilon}^{*}\left(\frac{d}{d x}\left(w \xi_{0}+\varepsilon Y\right)\right)=\varepsilon \xi_{\varepsilon}^{*}\left(T_{\varepsilon}^{\nu} Y\right)+\varepsilon \xi_{\varepsilon}^{*}(R) .
$$

Now using $\xi_{\varepsilon}^{*}\left(\xi_{0}\right)=\varepsilon$ and (4.31), we get

$$
\frac{d}{d x}\left\{w+a \mathcal{H}\left(w^{\prime}\right)+\xi_{\varepsilon}^{*}\left(\mathcal{T}_{\varepsilon, \nu}(R)\right)\right\}=\xi_{\varepsilon}^{*}\left(T_{\varepsilon}^{\nu} Y\right)+\xi_{\varepsilon}^{*}(R) .
$$

We observe that $\xi_{\varepsilon}^{*}(R) \in B_{3}^{\alpha}(\mathbb{R})$ is odd since $S R(x)=-R(-x)$ and $\xi_{\varepsilon}^{*}(S V)=\xi_{\varepsilon}^{*}(V)$. Therefore the following integral

$$
\int_{-\infty}^{x} \xi_{\varepsilon}^{*}(R(s)) d s
$$

is well defined in $B_{2}^{\alpha}(\mathbb{R})$. We now define $\mathcal{C}_{\varepsilon, \nu}(R)$ by

$$
\mathcal{C}_{\varepsilon, \nu}(R)=\int_{-\infty}^{x} \xi_{\varepsilon}^{*}\left(T_{\varepsilon}^{\nu} Y\right) d s-\xi_{\varepsilon}^{*}\left(\mathcal{T}_{\varepsilon, \nu}(R)\right) .
$$

It results from the estimates on $\mathcal{T}_{\varepsilon, \nu}(R)$ that

$$
\left\|\xi_{\varepsilon}^{*}\left(\mathcal{T}_{\varepsilon, \nu}(R)\right)\right\|_{2, \mathbb{R}}^{\alpha} \leq c(\varepsilon+|\nu||\ln \varepsilon|)\|R\|_{3, \widetilde{H}^{*}}^{\alpha} .
$$

Using the decomposition $Y=Y_{0}+Y_{1}$, the property (4.14), the decomposition (4.11) of $\Gamma_{\varepsilon, k}^{\nu}$, and Lemma A.3 of Appendix A for $Y_{0}$, and a lemma analogous to the lemma obtained for $\widehat{w}_{1}(k)$ in appendix B for $Y_{1}$, we prove that $\int_{-\infty}^{x}\left(\xi_{\varepsilon}^{*}\left(T_{\varepsilon}^{\nu} Y\right)\right) d s$ is in $B_{2}^{\alpha}(\mathbb{R})$ with

$$
\left\|\int_{-\infty}^{x} \xi_{\varepsilon}^{*}\left(T_{\varepsilon}^{\nu} Y\right) d s\right\|_{2, \mathbb{R}}^{\alpha} \leq|\nu| \varepsilon^{-1}\|R\|_{3, \mathbb{H}^{\cdot}}^{\alpha} .
$$

This leads to the estimate on $\mathcal{C}_{\varepsilon, \nu}(R)$ and concludes the proof of the lemma 4.4.

Remark for water wave examples: Notice that (4.32) corresponds to the differentiation of the Bernoulli first integral. For our explicit example of section 2, the Bernoulli first integral reads (see (2.5)) for $U$ decomposed as (4.1)

$$
w+\xi_{\varepsilon}^{*}(Y)-\frac{3-\varepsilon}{2} w^{2}+O(\varepsilon+|\nu|)=\text { const },
$$

where the coefficient of $w^{2}$ is $(a / 2) \xi_{\varepsilon}^{*}\left(D_{\varepsilon} \theta_{k}\right)$ (independent of $k$ ). Notice in addition for water wave examples that $\tau_{\varepsilon}^{\nu}(k)$ is independent of $k$. 


\subsubsection{Reduction}

We use Lemma 4.4 where we replace $R$ by $R_{\varepsilon}^{\nu}(w, Y)$. We then obtain

$$
Y=a \mathcal{T}(w)+\mathcal{T}_{\varepsilon}\left(R_{\varepsilon}^{\nu}(w, Y)\right) .
$$

and we now solve this equation in Theorem 4.9 for writing $Y$ as a function of $w, \nu$ and $\varepsilon$. Therefore (3.1) is finally reduced to an equation for $w$.

Theorem 4.9 (of reduction). Assume that $\mathcal{L}_{\varepsilon}$ satisfies hypotheses H1, H2, and H3, $\mathcal{N}_{\varepsilon}$ satisfies $H_{4}$ and $H 5$ and let $R_{\varepsilon}^{\nu}$ be defined by equation (4.2). Then for any $M>0$, there exists $\varepsilon_{0}>0$ and $\nu_{0}>0$ such that for $0<\varepsilon<\varepsilon_{0}, 0 \leq|\nu|<\nu_{0} \varepsilon$ and for all $w \in B_{2}^{1, \alpha}(\mathbb{R})$ such that $\|w\|_{2}^{1, \alpha} \leq M$, the reversible weak solutions $U \in \mathcal{B}^{\alpha}(\widehat{\mathbb{D}})$ of (3.1) written as $U=(\nu+w) \xi_{0}+\varepsilon Y$ must satisfy

$$
\begin{aligned}
Y & =Y_{\varepsilon, \nu}(w) \\
& =a \mathcal{T}(w)+O\left(\varepsilon+|\nu| \varepsilon^{-1}\right),
\end{aligned}
$$

where the mapping $Y_{\varepsilon, \nu}$ is smooth $B_{2}^{1, \alpha}(\mathbb{R}) \mapsto \mathcal{B}^{\alpha}(\widehat{\mathbb{D}})$. In the case when $\tau_{\varepsilon}^{\nu}(k)$ is independent of $k$, the above result is valid for $|\nu|<\nu_{0}$ and the estimate $O\left(\varepsilon+|\nu| \varepsilon^{-1}\right)$ is replaced by $O(\varepsilon+|\nu|)$.

This theorem corresponds to a reduction of the infinite dimensional dynamics to the study of the scalar function $w(x), Y$ being determined by $w, \varepsilon$ and $\nu$. Notice that H6 is not required in this Theorem.

Proof. Proposition 4.2 shows that if $U=(\nu+w) \xi_{0}+\varepsilon Y$ is symmetric then $R=$ $R_{\varepsilon}^{\nu}(w, Y)$ satisfies the hypothesis of lemma 4.4. Therefore we must find $Y \in \mathcal{B}^{\alpha}(\widehat{\mathbb{D}})$ symmetric such that

$$
Y-a \mathcal{T}(w)-\mathcal{J}(Y, w, \varepsilon, \nu)=0,
$$

where

$$
\mathcal{J}(Y, w, \varepsilon, \nu)=\mathcal{T}_{\varepsilon, \nu}\left(R_{\varepsilon}^{\nu}(w, Y)\right) .
$$

Here $w$ is fixed and is such that $\|w\|_{2, \mathbb{R}}^{1, \alpha} \leq M$. We solve this equation by using the implicit function theorem. From lemma 4.4 and proposition 4.2

$$
\|\mathcal{J}(Y, w, \varepsilon, \nu)\|_{\mathcal{B}} \leq c\left(\varepsilon+|\nu| \varepsilon^{-1}\right),
$$

holds for $\|Y\|_{\mathcal{B}} \leq \delta$. The same kind of estimate holds for the derivative of $\mathcal{J}(Y, w, \varepsilon, \nu)$ with respect to $Y$. Hence the differential of $Y-\mathcal{J}(Y, w, \varepsilon, \nu)$ at $Y=a \mathcal{T}(w)$ is close to identity. Actually we must adapt this theorem since we fix $\varepsilon$ small enough but not $0(\mathcal{J}$ is defined only for $\varepsilon>0)$. We then replace $\mathcal{J}(Y, w, \varepsilon, \nu)$ by

$$
\mathcal{J}(Y, w, \varepsilon, \nu)-\left(1-\mu \varepsilon^{-1}\right) \mathcal{J}(a \mathcal{T}(w), w, \varepsilon, \nu) .
$$

For $\mu=0$ we have the solution $Y=a \mathcal{T}(w)$, and equation (4.34) corresponds to $\mu=\varepsilon$. Applying the implicit function theorem for $\mu$ small and $Y$ near $a \mathcal{T}(w)$, we obtain the required result in making $\mu=\varepsilon$ which lies in the domain of existence of the solution. 


\subsubsection{Benjamin-Ono}

We now reduce the system (4.3)-(4.4) to a scalar equation. Indeed we already know that if $(w, Y)$ is a solution of (4.3)-(4.4) then $Y \in \mathcal{B}^{\alpha}(\widehat{\mathbb{D}})$ is given by $Y=Y_{\varepsilon, \nu}(w)=$ $a \mathcal{T}(w)+O\left(\varepsilon+|\nu| \varepsilon^{-1}\right)$ (Theorem 4.9). the following Lemma gives the equation satisfied by $w$.

Lemma 4.10 (equation for $\boldsymbol{w}$ ). Assume that $\mathcal{L}_{\varepsilon}$ satisfies hypothesis H1, H2, H3 and that $\mathcal{N}_{\varepsilon}$ satisfies hypotheses $H_{4}$ and H5. Let $U(x)=(\nu+w(x)) \xi_{0}+\varepsilon Y(x)$ be a reversible weak solution of system $(4.3)-(4.4)$ in $\mathcal{B}^{\alpha}(\widehat{\mathbb{D}})$, i.e. tending towards 0 at infinity. Then for $0<\varepsilon<\varepsilon_{0}$, and $|\nu|<\nu_{0} \varepsilon$, the function $w \in B_{2}^{1, \alpha}(\mathbb{R})$ satisfies

$$
w+a \mathcal{H}\left(\frac{d w}{d x}\right)+a c_{0} w^{2}=B_{\varepsilon, \nu}(w),
$$

where $B_{\varepsilon, \nu}: B_{2}^{1, \alpha}(\mathbb{R}) \rightarrow B_{2}^{\alpha}(\mathbb{R})$ is smooth and $O\left(\varepsilon+|\nu| \varepsilon^{-1}\right)$. In the case when $\tau_{\varepsilon}^{\nu}(k)$ is independent of $k$, the above result is valid for $|\nu|<\nu_{0}$ and the estimate $O\left(\varepsilon+|\nu| \varepsilon^{-1}\right)$ is replaced by $O(\varepsilon+|\nu|)$.

Notice that the left hand side of this equation is the Benjamin-Ono equation and the right hand side is a nice perturbation. We observe that hypothesis H5

$$
c_{\varepsilon, k}=\xi_{\varepsilon}^{*}\left(D_{\varepsilon} \theta_{k}\right)=2 c_{0}+\varepsilon \gamma_{\varepsilon}(k),
$$

involves two different kind of terms : on the one hand $\xi_{\varepsilon}^{*}$ and $\theta_{k}$ which come from the hypothesis on the linear operator $\mathcal{L}_{\varepsilon}$, and on the other hand the linear operator $D_{\varepsilon}$ which is part of the non linear operator $\mathcal{N}_{\varepsilon}$. Note that $a c_{0} w^{2}$ is the principal part of the non-local quadratic term

$$
a \int_{-\infty}^{x} u \frac{d}{d x} \mathcal{F}^{-1}\left(\widehat{u} c_{\varepsilon, k}\right)
$$

Proof of lemma 4.10. We already know with Theorem 4.9 that $Y=Y_{\varepsilon, \nu}(w)$. We then use (4.8) with $R=R_{\varepsilon}^{\nu}\left(w, Y_{\varepsilon, \nu}(w)\right)\left(R_{\varepsilon}^{\nu}\right.$ satisfies the hypothesis of lemma 4.4), hence

$$
w+a \mathcal{H}\left(w^{\prime}\right)=\int_{-\infty}^{x} \xi_{\varepsilon}^{*}\left(R_{\varepsilon}^{\nu}\left(w, Y_{\varepsilon, \nu}\right)\right) d s+\mathcal{C}_{\varepsilon, \nu}\left(R_{\varepsilon}^{\nu}\left(w, Y_{\varepsilon, \nu}(w)\right)\right) .
$$

Notice that from (4.30), we can write $Y_{\varepsilon, \nu}(w)$ as

$$
Y_{\varepsilon, \nu}(w)=a \mathcal{T}\left(w_{0}\right)+\widetilde{\mathcal{T}}_{\varepsilon, \nu}\left(R_{\varepsilon}^{\nu}\right),
$$

where $\widetilde{\mathcal{T}}_{\varepsilon, \nu}(R)=\mathcal{T}_{\varepsilon, \nu}(R)-a \mathcal{F}^{-1}\left(i k \widehat{w}_{1} \theta_{k}\right)$ satisfies the same estimate as $\mathcal{T}_{\varepsilon, \nu}(R)$ i.e.

$$
\left\|\widetilde{\mathcal{T}}_{\varepsilon, \nu}(R)\right\|_{\mathcal{B}} \leq c(\varepsilon+|\nu \| \ln \varepsilon|)\|R\|_{3, \widetilde{H}^{0}}^{\alpha} .
$$

Using the expression of $R_{\varepsilon}^{\nu}(w, Y)$ given by proposition 4.2 and the expression of $Y_{\varepsilon, \nu}(w)$ in (4.37) we obtain

$$
\begin{aligned}
R_{\varepsilon}^{\nu}\left(w, Y_{\varepsilon, \nu}(w)\right)= & a w D_{\varepsilon} \mathcal{T}\left(w_{0}\right)+w D_{\varepsilon} \widetilde{\mathcal{T}}_{\varepsilon, \nu}\left(R_{\varepsilon}^{\nu}\left(w, Y_{\varepsilon, \nu}(w)\right)\right) \\
& +\widetilde{R}_{\varepsilon}^{\nu}\left(w, Y_{\varepsilon, \nu}(w)\right)
\end{aligned}
$$


We also observe that

$$
\begin{aligned}
\xi_{\varepsilon}^{*}\left(D_{\varepsilon} \mathcal{T}\left(w_{0}\right)\right) & =\mathcal{F}^{-1}\left(-i k \widehat{w}_{0} c_{\varepsilon, k}\right) \\
& =-2 c_{0} w^{\prime}+2 c_{0} \mathcal{F}^{-1}\left(i k \varphi_{1} \widehat{w}\right)-\mathcal{F}^{-1}\left(i k \varphi_{0} \varepsilon \gamma_{\varepsilon}(k) \widehat{w}\right),
\end{aligned}
$$

where $c_{\varepsilon, k}=\xi_{\varepsilon}^{*}\left(D_{\varepsilon} \theta_{k}\right)=2 c_{0}+\varepsilon \gamma_{\varepsilon}(k)$. We can prove (using again Lemma A.2 with $\left.F_{\varepsilon}=\varepsilon \gamma_{\varepsilon}\right)$ that

$$
\begin{aligned}
\left\|\mathcal{F}^{-1}\left(i k \varphi_{0} \varepsilon \gamma_{\varepsilon}(k) \widehat{w}\right)\right\|_{2, \mathbb{R}}^{\alpha} & \leq c \varepsilon\left\|\mathcal{F}^{-1}(i k \widehat{w})\right\|_{2, \mathbb{R}}^{\alpha}, \\
& \leq c \varepsilon\|w\|_{2, \mathbb{R}}^{1, \alpha} .
\end{aligned}
$$

and we know from lemma 4.8 that

$$
\left\|\mathcal{F}^{-1}\left(i k \varphi_{1} \widehat{w}\right)\right\|_{2, \mathbb{R}}^{\alpha} \leq c \varepsilon\left\|R_{\varepsilon}^{\nu}\right\|_{2, \widetilde{H}}^{\alpha} \cdot
$$

We deduce that

$$
\int_{-\infty}^{x} \xi_{\varepsilon}^{*}\left(R_{\varepsilon}^{\nu}\left(w, Y_{\varepsilon, \nu}\right)\right) d s=-a c_{0} w^{2}+B_{\varepsilon, \nu, 0}(w),
$$

where

$$
\begin{aligned}
B_{\varepsilon, \nu, 0}(w)= & 2 a c_{0} \int_{-\infty}^{x} w \mathcal{F}^{-1}\left(i k \varphi_{1} \widehat{w}\right)-a \int_{-\infty}^{x} w \mathcal{F}^{-1}\left(i k \varphi_{0} \varepsilon \gamma_{\varepsilon}(k) \widehat{w}\right) \\
& +\int_{-\infty}^{x}\left[w \xi_{\varepsilon}^{*}\left(D_{\varepsilon} \widetilde{\mathcal{T}}_{\varepsilon}\left(R_{\varepsilon}^{\nu}\right)\right)+\xi_{\varepsilon}^{*}\left(\widetilde{R}_{\varepsilon}^{\nu}\right)\right] d s,
\end{aligned}
$$

for which the following holds, thanks to (4.40), (4.39)

$$
\left\|B_{\varepsilon, \nu, 0}(w)\right\|_{2, \mathbb{R}}^{\alpha} \leq c\left(\varepsilon+|\nu| \varepsilon^{-1}\right),
$$

for $\|w\|_{2, \mathbb{R}}^{1, \alpha} \leq M$.

We now define $B_{\varepsilon, \nu}(w)$ by

$$
B_{\varepsilon, \nu}(w)=B_{\varepsilon, \nu, 0}(w)+\mathcal{C}_{\varepsilon, \nu}\left(R_{\varepsilon}^{\nu}\left(w, Y_{\varepsilon, \nu}(w)\right)\right),
$$

which allows to derive the perturbed Benjamin - Ono equation (4.35).

As noticed above in our examples we obtain (4.35) quasi-directly from the Bernoulli first integral (see (4.33)).

\subsubsection{Bifurcation result}

The aim of this section is to find reversible solutions (i.e. even solutions) of (4.35) homoclinic to 0 in $B_{2}^{1, \alpha}(\mathbb{R})$. This will give reversible (weak) solutions of (3.1) homoclinic to 0 in $\mathcal{B}^{\alpha}(\widehat{\mathbb{D}})$, thanks to Theorem 4.9 . Next theorem shows the existence of an even solution of (4.35) approximated by

$$
u_{h}(x)=\frac{-2}{a c_{0}\left(1+(x / a)^{2}\right)},
$$

where it is known that $u_{h}$ is the unique non zero even solution of the Benjamin-Ono equation (see [2]), tending towards 0 at infinity

$$
u_{h}+a \mathcal{H}\left(\frac{d u_{h}}{d x}\right)+a c_{0} u_{h}^{2}=0 .
$$


Theorem 4.11. For $0<\varepsilon<\varepsilon_{0}$ and $|\nu|<\nu_{0} \varepsilon$, there is a unique reversible homoclinic solution $w \in B_{2}^{1, \alpha}(\mathbb{R})$ of (4.35) (perturbed Benjamin-Ono equation), such that

$$
\left\|w-u_{h}\right\|_{2, \mathbb{R}}^{1, \alpha} \leq c\left(\varepsilon+|\nu| \varepsilon^{-1}\right) .
$$

Collecting the results of Theorem 4.9, Lemma 4.10 and Theorem 4.11 ends the proof of Theorem 4.3.

Proof of Theorem 4.11. We again use the implicit function theorem starting from the approximate Benjamin - Ono homoclinic $u_{h}$. We look for $w \in B_{2}^{1, \alpha}(\mathbb{R})$ solution of the equation (4.35) under the form

$$
w=u_{h}+v .
$$

We need to find $v \in B_{2}^{1, \alpha}(\mathbb{R})$ satisfying

$$
L v=\mathcal{G}(v, \varepsilon)
$$

where

$$
L v=v+a \mathcal{H}\left(\frac{d v}{d x}\right)+2 a c_{0} u_{h} v
$$

and where $\mathcal{G}$ is defined by

$$
\mathcal{G}(v, \varepsilon)=B_{\varepsilon, \nu}\left(u_{h}+v\right)-a c_{0} v^{2} .
$$

The map $v \mapsto \mathcal{G}(v, \varepsilon)$ is in $\mathcal{C}^{k}\left(B_{2}^{1, \alpha}(\mathbb{R}), B_{2}^{\alpha}(\mathbb{R})\right)$, and (4.41) is invariant under the symmetry $\widehat{S}$

$$
v(x) \mapsto v(-x) .
$$

We need estimates on $\mathcal{G}(v, \varepsilon)$. By Lemma 4.10

$$
\|\mathcal{G}\|_{2, \mathbb{R}}^{\alpha} \leq c\left(\varepsilon+|\nu| \varepsilon^{-1}+\left(\|v\|_{2, \mathbb{R}}^{1, \alpha}\right)^{2}\right)
$$

for $\|v\|_{2, \mathbb{R}}^{1, \alpha} \leq \delta$. The same kind of estimate holds for the derivative of $\mathcal{G}$ with respect to $v$. Then in a sufficiently small ball for $v \in B_{2}^{1, \alpha}(\mathbb{R})$ the differential at the origin of the operator $L v-\mathcal{G}(v, \varepsilon)$ is close to the operator $L$ which has a bounded inverse in $\mathcal{L}\left(B_{2}^{\alpha}(\mathbb{R}), B_{2}^{1, \alpha}(\mathbb{R})\right)$ in the subset of even functions, thanks to a result of [1]. Actually we need to adapt the implicit function theorem as in the proof of Theorem 4.9, since we fix $\varepsilon$ small enough, but not 0 ( $\mathcal{G}$ is not defined for $\varepsilon=0$ ). Hence we replace $\mathcal{G}(v, \varepsilon)$ by

$$
\mathcal{G}(v, \varepsilon)-\left(1-\mu \varepsilon^{-1}\right) \mathcal{G}(0, \varepsilon),
$$

and consider the implicit function theorem for $(v, \mu)$ near 0 . For $\mu=0$ we have the trivial solution $v=0$. Equation (4.41) corresponds to $\mu=\varepsilon$, which lies in the domain of existence of the solution obtained by the implicit function theorem, for $\varepsilon$ small enough. Finally we obtain

$$
\|v\|_{2, \mathbb{R}}^{1, \alpha}=O\left(\varepsilon+|\nu| \varepsilon^{-1}\right),
$$

and theorem 4.11 is proved. 


\section{A Proof of lemma $4.6(\varepsilon|k|<\delta)$}

We first give a technical lemma which will be useful in Appendix A and B (see [7] lemma B1 p.2314).

Lemma A.1. Let $\mathbb{E}$ and $\mathbb{F}$ be Banach spaces and assume $K: \mathbb{R} \rightarrow \mathcal{L}(\mathbb{E}, \mathbb{F})$ is a function $\mathcal{C}^{1}$ on $\mathbb{R} \backslash\{0\}$ such that

i) $\|K(x)\|_{\mathcal{L}(\mathbb{E}, \mathbb{F})} \leq C_{0} /|x|,\left\|K^{\prime}(x)\right\|_{\mathcal{L}(\mathbb{E}, \mathbb{F})} \leq C_{0} /|x|^{2}$ for $|x| \leq 1$,

ii) $\|K(x)\|_{\mathcal{L}(\mathbb{E}, \mathbb{F})} \leq C_{1} /|x|^{2}$ for $|x| \geq 1$, and p.v. $\int_{-1}^{1} K(x) d x \in \mathcal{L}(\mathbb{E}, \mathbb{F})$.

Then the linear map $\mathcal{K}$ defined by

$$
f \mapsto \mathcal{K} f=p . v \cdot \int_{\mathbb{R}} K(s) f(.-s) d s,
$$

is bounded from $B_{2}^{\alpha}(\mathbb{E})$ to $B_{2}^{\alpha}(\mathbb{F})$.

With lemma A.1 we prove the following lemmas A.2 and A.3. These lemmas allow us to obtain the estimates of lemma 4.6.

Lemma A.2. Let $\mathbb{E}$ and $\mathbb{F}$ be Banach spaces and let $F_{\varepsilon}(k) \in \mathcal{L}(\mathbb{E}, \mathbb{F})$ such that $k \mapsto F_{\varepsilon}(k) \in \mathcal{C}_{\lim }^{2}(\mathbb{R}, \mathcal{L}(\mathbb{E}, \mathbb{F}))$, and let $R \in B_{2}^{\alpha}(\mathbb{E})$. Then

$$
\mathcal{M}_{\varepsilon}^{p}(R)(x)=\mathcal{F}^{-1}\left(\frac{\varphi_{0}(\varepsilon k)}{\Delta^{p}} F_{\varepsilon}(k)(\widehat{R})\right) \in B_{2}^{1, \alpha}(\mathbb{F}), p=0,1,2,3,
$$

with the estimate

$$
\begin{aligned}
(a)\left\|\mathcal{M}_{\varepsilon}^{p}(R)\right\|_{2, \mathbb{F}}^{\alpha} \leq & c\left(\left\|F_{\varepsilon}\right\|+\varepsilon^{\min (p-1,0)}|\ln \varepsilon|^{\delta_{p, 1}}\left\|F_{\varepsilon}^{\prime}\right\|\right. \\
& \left.+\varepsilon^{\min (p-2,0)}|\ln \varepsilon|^{\delta_{p, 2}}\left\|F_{\varepsilon}^{\prime \prime}\right\|\right)\|R\|_{2, \mathbb{E}}^{\alpha}, \\
(b)\left\|\frac{d \mathcal{M}_{\varepsilon}^{p}(R)}{d x}\right\|_{2, \mathbb{F}}^{\alpha} \leq & c\left(\left\|F_{\varepsilon}\right\|+\varepsilon^{\min (p-2,0)}|\ln \varepsilon|^{\delta_{p, 2}}\left\|F_{\varepsilon}^{\prime}\right\|\right. \\
& \left.+\varepsilon^{\min (p-3,0)}|\ln \varepsilon|^{\delta_{p, 3}}\left\|F_{\varepsilon}^{\prime \prime}\right\|\right)\|R\|_{2, \mathbb{E}}^{\alpha},
\end{aligned}
$$

where $\left\|F_{\varepsilon}^{(n)}\right\|=\sup _{0<\varepsilon|k|<\delta}\left\|F_{\varepsilon}^{(n)}(k)\right\|_{\mathcal{L}(\mathbb{E}, \mathbb{F})}$ for $n=0,1,2$, and $\delta_{i, j}=0$ for $i \neq j$ and $\delta_{i, i}=1$.

Moreover, if $k \mapsto F_{\varepsilon}(k)$ is even but not continuous in $k=0$ then the same conclusions hold.

Proof. (a) We define for $p=0,1,2,3$, the operator $K_{p}(x)$ in $\mathcal{L}(\mathbb{E}, \mathbb{F})$ by

$$
K_{p}(x) . V=\mathcal{F}^{-1}\left(\frac{\varphi_{0}}{\Delta^{p}} F_{\varepsilon}(k)(V)\right),
$$

and we prove that $K_{p}$ satisfies the assumptions of lemma A.1. In the case when $F_{\varepsilon}$ is even $K_{p}(x)$ reads

$$
K_{p, 1}(x)=2 \int_{0}^{\delta / \varepsilon} \frac{\varphi_{0}(\varepsilon k)}{\Delta(\varepsilon, k)^{p}} F_{\varepsilon}(k) \cos (k x) d k,
$$


then after two integrations by parts $K_{p, 1}$ can be written as

$$
K_{p, 1}(x)=-2 \int_{0}^{\delta / \varepsilon}\left(\frac{\varphi_{0} F_{\varepsilon}}{\Delta^{p}}\right)^{\prime} \frac{\sin (k x)}{x} d k
$$

and

$$
K_{p, 1}(x)=2 \frac{-a p F_{\varepsilon}(0)+F_{\varepsilon}^{\prime}(0)}{x^{2}}-2 \int_{0}^{\delta / \varepsilon}\left(\frac{\varphi_{0} F_{\varepsilon}}{\Delta^{p}}\right)^{\prime \prime} \frac{\cos (k x)}{x^{2}} d k .
$$

We now differentiate (A.2) and perform two integrations by parts so that $K_{p, 1}^{\prime}(x)$ reads

$$
K_{p, 1}^{\prime}(x)=2 \int_{0}^{\delta / \varepsilon}\left(\frac{k}{\Delta^{p}} \varphi_{0} F_{\varepsilon}\right)^{\prime \prime} \frac{\sin (k x)}{x^{2}} d k
$$

An integration of (A.2) gives the following relation

$$
p . v . \int_{-1}^{1} K_{p, 1}(x) d x=4 \int_{0}^{\delta / \varepsilon} \varphi_{0} F_{\varepsilon} \frac{\sin (k)}{k \Delta^{p}} d k .
$$

After straightforward estimations, (A.3), (A.4), (A.5) and (A.6) allow to satisfy the assumptions of lemma A.1 where the dependency of the constants in $\varepsilon$ and $\left\|F_{\varepsilon}^{(n)}\right\|$, for $n=0,1,2$ follows. Then, the part (a) of lemma A.2 is proved for $F_{\varepsilon}$ even. Notice that the continuity of $F_{\varepsilon}$ in $k=0$ is not required in this case.

In the case when $F_{\varepsilon}$ is odd we use the same strategy, the only difference is in the first integration by parts

$$
K_{p, 2}(x)=-2 \frac{F_{\varepsilon}(0)}{x}+2 \int_{0}^{\delta / \varepsilon}\left(\frac{\varphi_{0}}{\Delta^{p}} F_{\varepsilon}\right)^{\prime} \frac{\cos (k x)}{x} d k .
$$

Here we use the continuity of $F_{\varepsilon}$, in particular the fact that $F_{\varepsilon}(0)=0$ so that we find an expression similar to the one found in the previous case, then we obtain the same estimate as $K_{p, 1}$, and the part (a) of lemma A.2 is proved for $F_{\varepsilon}$ odd.

(b) The proof of (b) is similar to the previous case, defining an operator $\widetilde{K}_{p}(x)$ in $\mathcal{L}(\mathbb{E}, \mathbb{F})$ by

$$
\widetilde{K}_{p}(x) . V=\mathcal{F}^{-1}\left(\frac{k \varphi_{0}}{\Delta^{p}} F_{\varepsilon}(k)(V)\right) .
$$

The same study as in (a) allows to satisfy the assumptions of lemma A.1 with the dependency of the constants in $\varepsilon$ and $\left\|F_{\varepsilon}^{(n)}\right\|$, for $n=0,1,2$ indicated in the lemma A.2.

In the next Lemma we study the primitives of the functions introduced in Lemma A.2.

Lemma A.3. Let $F_{\varepsilon}(k) \in \mathcal{L}(\mathbb{E}, \mathbb{F})$ such that $F_{\varepsilon}(k) S=F_{\varepsilon}(-k)$ and $k \mapsto F_{\varepsilon}(k) \in$ $\mathcal{C}_{\lim }^{3}(\mathbb{R}, \mathcal{L}(\mathbb{E}, \mathbb{F}))$, and let $R \in B_{3}^{\alpha}(\mathbb{E})$ such that $S R(x)=-R(-x)$. Then

$$
\mathcal{P}_{\varepsilon}^{p}(R)(x)=\mathcal{F}^{-1}\left(\frac{\varphi_{0}(\varepsilon k)}{i k \Delta^{p}} F_{\varepsilon}(k)(\widehat{R})\right) \in B_{2}^{\alpha}(\mathbb{F}), p=0,1,2,3
$$


with the estimate

$$
\begin{aligned}
\left\|\mathcal{P}_{\varepsilon}^{p}(R)\right\|_{2, \mathbb{F}}^{\alpha} \leq & c\left(\left\|F_{\varepsilon}\right\|+\left\|F_{\varepsilon}^{\prime}\right\|\right)\|R\|_{3, \mathbb{E}}^{\alpha}+ \\
& +c\left(\varepsilon^{\min (p-1,0)}|\ln \varepsilon|^{\delta_{p, 1}}\left\|F_{\varepsilon}^{\prime \prime}\right\|+\varepsilon^{\min (p-2,0)}|\ln \varepsilon|^{\delta_{p, 2}}\left\|F_{\varepsilon}^{(3)}\right\|\right)\|R\|_{2, \mathbb{E}}^{\alpha},
\end{aligned}
$$

where $\left\|F_{\varepsilon}^{(n)}\right\|=\sup _{0<\varepsilon|k|<\delta}\left\|F_{\varepsilon}^{(n)}(k)\right\|_{\mathcal{L}(\mathbb{E}, \mathbb{F})}$ for $n=0,1,2,3$.

Proof. We first decompose $\mathcal{P}_{\varepsilon}^{p}(R)$ as

$$
\mathcal{P}_{\varepsilon}^{p}(R)=\mathcal{F}^{-1}\left(\frac{\varphi_{0}}{i k \Delta^{p}} F_{\varepsilon}(0)(\widehat{R})\right)+\mathcal{F}^{-1}\left(\frac{\varphi_{0}}{\Delta^{p}} J_{\varepsilon}(k)(\widehat{R})\right),
$$

where we define $J_{\varepsilon}$ by

$$
J_{\varepsilon}(k)(V)=\frac{F_{\varepsilon}(k)(V)-F_{\varepsilon}(0)(V)}{i k} .
$$

We study the first term of (A.7) which can be written as

$$
\mathcal{F}^{-1}\left(\frac{\varphi_{0}}{i k \Delta^{p}} F_{\varepsilon}(0)(\widehat{R})\right)=K_{p, 3}(x) * \sigma(x)
$$

where

$$
K_{p, 3}(x)=\mathcal{F}^{-1}\left(\frac{\varphi_{0}}{\Delta^{p}}\right), \quad \sigma(x)=\mathcal{F}^{-1}\left(\frac{F_{\varepsilon}(0)(\widehat{R})}{i k}\right) .
$$

Since $R \in B_{3}^{\alpha}(\mathbb{E})$ satisfies $S R(x)=-R(-x)$ and $F_{\varepsilon}(0)(S V)=F_{\varepsilon}(0)(V)$ then the map $x \mapsto F_{\varepsilon}(0)(R(x))$ is odd and the function

$$
\sigma(x)=\int_{-\infty}^{x} F_{\varepsilon}(0)(R(s)) d s,
$$

is an even primitive of $F_{\varepsilon}(0)(R)$ and is in $B_{2}^{1, \alpha}(\mathbb{F})$ with

$$
\|\sigma\|_{2, \mathbb{F}}^{1, \alpha} \leq c\left\|F_{\varepsilon}\right\|\|R\|_{3, \mathbb{E}}^{\alpha} .
$$

An estimate of the operator $K_{p, 3}(x)$ is obtained from lemma A.2 in using $F_{\varepsilon}=1$. We conclude that

$$
\left\|\mathcal{F}^{-1}\left(\frac{\varphi_{0}}{i k \Delta^{p}} F_{\varepsilon}(0)(\widehat{R})\right)\right\|_{2, \mathbb{F}}^{\alpha} \leq c\left\|F_{\varepsilon}\right\|\|R\|_{3, \mathbb{E}}^{\alpha} .
$$

Now, we want to use Lemma A.2 to estimate $\mathcal{F}^{-1}\left(\frac{\varphi_{0}}{\Delta^{p}} J_{\varepsilon}(k)(\widehat{R})\right)$ but the map $J_{\varepsilon}$ is not necessarily continuous in $k=0$. Then we study separately the cases $F_{\varepsilon}$ even or odd.

Assume $\boldsymbol{F}_{\varepsilon}$ is even. In this case we write

$$
\begin{aligned}
\mathcal{F}^{-1}\left(\frac{\varphi_{0}}{\Delta^{p}} J_{\varepsilon}(k)(\widehat{R})\right)= & \mathcal{F}^{-1}\left(\frac{\varphi_{0}}{\Delta^{p}}\left\{J_{\varepsilon}(k)+i \operatorname{sgn}(k) F_{\varepsilon}^{\prime}\left(0^{+}\right)\right\}(\widehat{R})\right) \\
& -\mathcal{F}^{-1}\left(\frac{\varphi_{0}}{\Delta^{p}} i \operatorname{sgn}(k) F_{\varepsilon}^{\prime}\left(0^{+}\right)(\widehat{R})\right) .
\end{aligned}
$$

The map $k \mapsto J_{\varepsilon}(k)+i \operatorname{sgn}(k) F_{\varepsilon}^{\prime}\left(0^{+}\right)$is in $\mathcal{C}_{\lim }^{2}(\mathbb{R}, \mathcal{L}(\mathbb{E}, \mathbb{F}))$ then Lemma A.2 applies to the first term of the r.h.s of (A.9). To estimate the second term, we write

$$
\mathcal{F}^{-1}\left(\frac{\varphi_{0}}{\Delta^{p}} i \operatorname{sgn}(k) F_{\varepsilon}^{\prime}\left(0^{+}\right)(\widehat{R})\right)=K_{p, 3}(x) * \mathcal{H}\left(\omega^{\prime}\right),
$$


where $K_{p}$ is the function introduced in (A.8), $\mathcal{H}$ is the Hilbert transform and where

$$
\omega(x)=\int_{-\infty}^{x} F_{\varepsilon}^{\prime}\left(0^{+}\right)(R(s)) d s,
$$

since $F_{\varepsilon}(k) S=F_{\varepsilon}(-k)$ then $F_{\varepsilon}^{\prime}\left(0^{+}\right) S=-F_{\varepsilon}^{\prime}\left(0^{-}\right)$, and $F_{\varepsilon}^{\prime}\left(0^{+}\right) S=F_{\varepsilon}^{\prime}\left(0^{+}\right)$because $F_{\varepsilon}$ is even. Moreover $R \in B_{3}^{\alpha}(\mathbb{E})$ satisfies $S R(x)=-R(-x)$ then $\omega$ is even and in $B_{2}^{1, \alpha}(\mathbb{F})$ with

$$
\|\omega\|_{2, \mathbb{F}}^{1, \alpha} \leq c\left\|F_{\varepsilon}^{\prime}\right\|\|R\|_{3, \mathbb{E}}^{\alpha} .
$$

We deduce that $\mathcal{H}\left(\omega^{\prime}\right) \in B_{2}^{\alpha}(\mathbb{F})$ (see [7], corollary B2 p.2317) and finally, as above, that

$$
\left\|\mathcal{F}^{-1}\left(\frac{\varphi_{0}}{\Delta^{p}} i \operatorname{sgn}(k) F_{\varepsilon}^{\prime}\left(0^{+}\right)(\widehat{R})\right)\right\|_{2, \mathbb{F}}^{\alpha} \leq c\left\|F_{\varepsilon}^{\prime}\right\|\|R\|_{3, \mathbb{E}}^{\alpha}
$$

holds. This ends the study of $\mathcal{F}^{-1}\left(\frac{\varphi_{0}}{\Delta^{p}} J_{\varepsilon}(k)(\widehat{R})\right)$ in the case when $F_{\varepsilon}$ is even.

Assume $\boldsymbol{F}_{\varepsilon}$ is odd. In this case $J_{\varepsilon}$ is even and Lemma A.2 applies, which ends the proof of Lemma A.3

\section{B Proof of Lemma $4.8(\varepsilon|k|>\delta)$}

Here we prove estimates necessary for the control of $w_{1}$ and $Y_{1}$ defined by (4.27) and (4.28).

Proposition B.1. a) Let $F_{\varepsilon}$ be a linear map where $k \mapsto F_{\varepsilon}(k)$ is continuously differentiable in $\mathcal{L}(\mathbb{H}, \mathbb{D})$ for $\varepsilon|k| \geq \delta / 2$ with the following estimates in $\mathcal{L}(\widetilde{\mathbb{H}}, \mathbb{H})$ and in $\mathcal{L}(\widetilde{\mathbb{H}}, \widehat{\mathbb{D}})(\varepsilon|k| \geq \delta / 2)$

$$
\begin{gathered}
\left\|\partial_{k}^{n} F_{\varepsilon}\right\|_{\mathcal{L}(\widetilde{\mathbb{H}}, \mathbb{H})} \leq \frac{c}{|k|^{n+1}}, n=0,1,2,3 \\
\left\|\partial_{k}^{n} F_{\varepsilon}\right\|_{\mathcal{L}(\widetilde{\mathbb{H}}, \widehat{\mathbb{D}})} \leq \frac{c \varepsilon}{|k|^{n}}, n=0,1,2,3 .
\end{gathered}
$$

Then the linear map

$$
V \rightarrow K_{0}(x) . V=\mathcal{F}^{-1}\left(F_{\varepsilon}(k)(V) \varphi_{1}(\varepsilon k)\right),
$$

satisfies the hypothesis of lemma $A .1$ in $\mathcal{L}(\widetilde{\mathbb{H}}, \widehat{\mathbb{D}})$, with constants of order $\varepsilon$.

b) the linear forms $\varepsilon p_{0}^{*} K_{0}(x)$ and $\frac{d}{d x} \varepsilon p_{0}^{*} K_{0}(x)$ satisfy the hypothesis of lemma A.1 in $\widetilde{\mathbb{H}}^{*}$, with constants of order $\varepsilon$.

We deduce from proposition B.1 and lemma A.1 the corollary

Corollary B.2. Let $F_{\varepsilon}$ satisfy the assumptions of proposition B.1, and let $R \in$ $B_{2}^{\alpha}(\widetilde{\mathbb{H}})$. Then $\mathcal{F}^{-1}\left(\varphi_{1} F_{\varepsilon}(\widehat{R})\right) \in B_{2}^{\alpha}(\widehat{\mathbb{D}})$ and $\mathcal{F}^{-1}\left(p_{0}^{*}\left(\varepsilon \varphi_{1} F_{\varepsilon}(\widehat{R})\right)\right) \in B_{2}^{1, \alpha}(\mathbb{R})$ with

$$
\begin{gathered}
\left\|\mathcal{F}^{-1}\left(\varphi_{1} F_{\varepsilon}(\widehat{R})\right)\right\|_{2, \widehat{\mathbb{D}}}^{\alpha} \leq c \varepsilon\|R\|_{2, \widetilde{\mathbb{H}}}^{\alpha}, \\
\left\|\mathcal{F}^{-1}\left(p_{0}^{*}\left(\varepsilon \varphi_{1} F_{\varepsilon}(\widehat{R})\right)\right)\right\|_{2, \mathbb{R}}^{1, \alpha} \leq c \varepsilon\|R\|_{2, \widetilde{\mathbb{H}}}^{\alpha}
\end{gathered}
$$


Proof. a) We first suppose that $F_{\varepsilon}$ is even, $K_{0}$ is then denoted by $K_{01}$. Since $\left\|F_{\varepsilon}\right\|_{\mathcal{L}(\widetilde{\mathbb{H}}, \mathbb{H})} \leq \frac{c}{k}, K_{01}$ may be written in $L^{2}(\mathbb{R}, \mathcal{L}(\widetilde{\mathbb{H}}, \mathbb{H}))$ as

$$
K_{01}(x)=2 \lim _{A \rightarrow \infty} \int_{\delta / 2 \varepsilon}^{A} \varphi_{1} F_{\varepsilon} \cos (k x) d k .
$$

After an integration by parts in the above integral, the limit can be computed in $L^{2}(\mathbb{R}, \mathcal{L}(\widetilde{\mathbb{H}}, \mathbb{H}))$ so that $K_{01}$ can be decomposed as follows

$$
\frac{1}{2} K_{01}(x)=-I_{1}(x)-\varepsilon I_{2}(x),
$$

where

$$
I_{1}(x)=\int_{\delta / 2 \varepsilon}^{\infty} F_{\varepsilon}^{\prime}(k) \frac{\sin (k x)}{x} \varphi_{1} d k
$$

and

$$
I_{2}(x)=\int_{\delta / 2 \varepsilon}^{\delta / \varepsilon} F_{\varepsilon}(k) \frac{\sin (k x)}{x} \varphi_{1}^{\prime} d k .
$$

Notice that $I_{2} \in \mathcal{C}^{0}(\mathbb{R}, \mathcal{L}(\widetilde{\mathbb{H}}, \widehat{\mathbb{D}}))$ and satisfies

$$
\left\|I_{2}(x)\right\|_{\mathcal{L}(\widetilde{\mathbb{H}}, \widehat{\mathbb{D}})} \leq \int_{\delta / 2 \varepsilon}^{\delta / \varepsilon} \varepsilon \frac{|\sin (k x)|}{|x|} d k \leq \frac{c}{|x|} .
$$

We also observe that after an integration by parts, the following estimate holds for $I_{2}$

$$
\left\|I_{2}(x)\right\|_{\mathcal{L}(\widetilde{\mathbb{H}}, \widehat{\mathbb{D}})} \leq \int_{\delta / 2 \varepsilon}^{\delta / \varepsilon} \frac{|\cos (k x)|}{x^{2}}\left(\frac{\varepsilon}{k}+\varepsilon^{2}\right) d k \leq \frac{c \varepsilon}{x^{2}}
$$

Let us now compute the derivative of $I_{2}$

$$
I_{2}^{\prime}(x)=-\frac{1}{x^{2}} \int_{\delta / 2 \varepsilon}^{\delta / \varepsilon} F_{\varepsilon}(k) \sin (k x) \varphi_{1}^{\prime} d k+\int_{\delta / 2 \varepsilon}^{\delta / \varepsilon} k F_{\varepsilon}(k) \frac{\cos (k x)}{x} \varphi_{1}^{\prime} d k .
$$

After an integration by parts in the second integral of (B.4), we obtain the following estimate

$$
\left\|I_{2}^{\prime}(x)\right\|_{\mathcal{L}(\widetilde{\mathbb{H}, \mathbb{\mathbb { D }}})} \leq \frac{c}{x^{2}} .
$$

Finally, we check

$$
\text { p.v. } \int_{-1}^{1} I_{2}(x) d x=2 \int_{\delta / 2 \varepsilon}^{\delta / \varepsilon} F_{\varepsilon}(k) \varphi_{1}^{\prime} \int_{0}^{k} \frac{\sin (u)}{u} d u d k=O(1) .
$$

The estimates (B.2), (B.3), (B.5) and (B.6) correspond to the hypothesis of Lemma A.1 for $I_{2}$. To end the proof of proposition B.1 a) in the case $F_{\varepsilon}$ even, we need to study $I_{1}$ which, after an integration by parts, and using $\left\|F_{\varepsilon}^{\prime \prime}\right\|_{\mathcal{L}(\widetilde{\mathbb{H}}, \mathbb{\mathbb { D }})} \leq \frac{\varepsilon}{k^{2}}$ and $\left\|F_{\varepsilon}^{\prime}\right\|_{\mathcal{L}(\widetilde{\mathbb{H}}, \widehat{\mathbb{D}})} \leq \frac{\varepsilon}{k}$, leads to

$$
\left\|I_{1}(x)\right\|_{\mathcal{L}(\widetilde{\mathbb{H}}, \widehat{\mathbb{D}})} \leq c \varepsilon \min \left(\frac{1}{|x|}, \frac{\varepsilon}{|x|^{2}}\right)
$$


We now need to compute the derivative of $I_{1}$. We first observe that

$$
I_{1}(x)=\int_{\delta / 2 \varepsilon}^{\infty}\left(F_{\varepsilon}^{\prime}(k) \varphi_{1}\right)^{\prime \prime} \frac{\sin (k x)-k x}{x^{3}} d k,
$$

so that $I_{1}^{\prime}$ reads

$$
\begin{aligned}
I_{1}^{\prime}(x)= & \int_{\delta / 2 \varepsilon}^{\infty} F_{\varepsilon}^{\prime \prime \prime}(k) \varphi_{1} h(k, x) d k+2 \varepsilon \int_{\delta / 2 \varepsilon}^{\delta / \varepsilon} F_{\varepsilon}^{\prime \prime}(k) \varphi_{1}^{\prime} h(k, x) d k+ \\
& +\varepsilon^{2} \int_{\delta / 2 \varepsilon}^{\delta / \varepsilon} F_{\varepsilon}^{\prime}(k) \varphi_{1}^{\prime \prime} h(k, x) d k
\end{aligned}
$$

where

$$
h(k, x)=(k x \cos (k x)-3 \sin (k x)+2 k x) / x^{4} .
$$

We deduce the following estimate

$$
\left\|I_{1}^{\prime}(x)\right\|_{\mathcal{L}(\widetilde{\mathbb{H}, \widehat{\mathbb{D}})}} \leq \frac{c \varepsilon}{x^{2}}
$$

finally we check

$$
p . v . \int_{-1}^{1} I_{1}(x) d x=2 \int_{\delta / 2 \varepsilon}^{\infty} F_{\varepsilon}^{\prime}(k) \varphi_{1} \int_{0}^{k} \frac{\sin (u)}{u} d u d k=O(\varepsilon) .
$$

The estimates (B.7), (B.8) and (B.9) correspond to the hypothesis of lemma A.1 then proposition B.1 a) is proved when $F_{\varepsilon}$ is even. We obtain the same results when $F_{\varepsilon}$ is odd with a similar proof.

We now prove proposition B.1 b). We need to study $\widetilde{K}_{0}=\mathcal{F}^{-1}\left(\varepsilon p_{0}^{*}\left(F_{\varepsilon}(k)\right) \varphi_{1}\right)$, where $F_{\varepsilon}$ behaves like $1 / k$ in $\mathcal{L}(\mathbb{H})$. We prove as for $K_{01}$ that when $F_{\varepsilon}$ is even

$$
\frac{1}{2} \widetilde{K}_{01}(x)=-\varepsilon \int_{\delta / 2 \varepsilon}^{\infty} p_{0}^{*}\left(F_{\varepsilon}^{\prime}(k)\right) \frac{\sin (k x)}{x} \varphi_{1} d k-\varepsilon^{2} \int_{\delta / 2 \varepsilon}^{\delta / \varepsilon} p_{0}^{*}\left(F_{\varepsilon}(k)\right) \frac{\sin (k x)}{x} \varphi_{1}^{\prime} d k
$$

holds, which leads to the estimate

$$
\left\|\widetilde{K}_{01}(x)\right\|_{\mathbb{H}^{*}} \leq \frac{c \varepsilon}{|x|},
$$

and

$$
p . v . \int_{-1}^{1} \widetilde{K}_{01}(x) d x=O(\varepsilon) .
$$

After integrations by parts we obtain

$$
\left\|\widetilde{K}_{01}(x)\right\|_{\mathbb{H}^{*}} \leq \frac{c \varepsilon}{|x|^{2}}
$$

and in differentiating $\widetilde{K}_{01}$ as follows

$$
\frac{d \widetilde{K}_{01}(x)}{d x}=2 \varepsilon \int_{\delta / 2 \varepsilon}^{\infty} \frac{\left(p_{0}^{*}\left(F_{\varepsilon}(k)\right) \varphi_{1}(\varepsilon k)\right)^{\prime \prime}}{x^{3}}(2 \cos (k x)-2+k x \sin (k x)) d k,
$$


it results

$$
\left\|\frac{d \widetilde{K}_{01}(x)}{d x}\right\|_{\mathbb{H}^{*}} \leq c \varepsilon \int_{\delta / 2 \varepsilon}^{\infty} \frac{|g(k x)|}{k^{3} x^{3}} d k+c \varepsilon^{2} \int_{\delta / 2 \varepsilon}^{\delta / \varepsilon} \frac{|g(k x)|}{k^{2} x^{3}} d k+c \varepsilon^{3} \int_{\delta / 2 \varepsilon}^{\delta / \varepsilon} \frac{|g(k x)|}{k x^{3}} d k,
$$

where

$$
g(u)=u \sin (u)-2+2 \cos (u) .
$$

Therefore using $g(u) / u^{3} \leq c \min \left(u, 1 / u^{2}\right), g(u) / u^{2} \leq c$ and $g(u) / u \leq u$ in the previous estimate, we obtain

$$
\left\|\frac{d \widetilde{K}_{01}(x)}{d x}\right\|_{\mathbb{H}^{*}} \leq \frac{c \varepsilon}{|x|}
$$

Similarly we obtain

$$
\begin{aligned}
\left\|\frac{d \widetilde{K}_{01}(x)}{d x}\right\|_{\mathbb{H}^{*}} & \leq c \varepsilon \int_{\delta / 2 \varepsilon}^{\infty} \frac{|\sin (k x)|}{k^{2} x^{2}} d k+c \varepsilon^{2} \int_{\delta / 2 \varepsilon}^{\delta / \varepsilon} \frac{|\sin (k x)|}{k x^{2}} d k+\varepsilon^{3} \int_{\delta / 2 \varepsilon}^{\delta / \varepsilon} \frac{|\sin (k x)|}{x^{2}} d k \\
& \leq \frac{c \varepsilon}{|x|^{2}} .
\end{aligned}
$$

To compute $d^{2} \widetilde{K}_{01} / d x^{2}$ we make an integration by parts in (B.13), then we obtain

$$
\frac{d^{2} \widetilde{K}_{01}}{d x^{2}}=\varepsilon \int_{\delta / 2 \varepsilon}^{\infty}\left(p_{0}^{*}\left(F_{\varepsilon}(k)\right) \varphi_{1}(\varepsilon k)\right)^{(3)} \frac{f(k x)}{x^{5}} d k
$$

where

$$
f(u)=-6 u-6 u \cos (u)-u^{2} \sin (u)+12 \sin (u) .
$$

We obtain as for (B.15) the estimate

$$
\left\|\frac{d^{2} \widetilde{K}_{01}}{d x^{2}}\right\|_{\mathbb{H}^{*}} \leq \frac{c \varepsilon}{|x|^{2}}
$$

Finally, we check

$$
\text { p.v. } \int_{-1}^{1} \frac{d \widetilde{K}_{01}}{d x} d x=0 .
$$

The estimates (B.10), (B.11), (B.12), (B.14), (B.15), (B.16) and (B.17) correspond to the hypothesis of Lemma A.1 for $\widetilde{K}_{01}$ and $\partial_{x} \widetilde{K}_{01}$, then b) is proved for $F_{\varepsilon}$ even. Similarly we obtain the same results when $F_{\varepsilon}$ is odd and proposition B.1 b) is proved.

\section{References}

[1] C. Amick, On the Theory of Internal Waves of Permanent Form in Fluids of great Depth, Trans. Amer. Math. Soc. 364 (1994) 399-419

[2] C. Amick, J. Toland, Uniqueness and Related Analytic Properties for the Benjamin-Ono Equation - a Nonlinear Neumann Problem in the Plane, Acta Math. 105 (1989) 1-49 
[3] T. B. Benjamin, Internal Waves of Permanent Form in Fluids of Great Depth, J. Fluid Mech. 29 (1967) 559-592

[4] F. Dias, G. Iooss, Water-Waves as a Spatial Dynamical System, Handbook of Mathematical Fluid Dynamics, vol II, S. Friedlander, D. Serre Eds, Elsevier (2003) 443-499

[5] G. Iooss, Gravity and Capillary-Gravity Periodic Traveling Waves for Two Superposed Fluid Layers, one Being of Infinite Depth, J. Math. Fluid. Mech. 1 (1999) 24-61

[6] G. Iooss, P. Kirrmann, Capillary Gravity Waves on the Free Surface of an Inviscid Fluid of Infinite Depth. Existence of Solitary Waves. Arch. Rat. Mech. Anal. 136 (1996) 1-19

[7] G. Iooss, E. Lombardi, S.M. Sun, Gravity Traveling Waves for Two Superposed Fluid Layers of infinite depth : a new type of bifurcation, Phil. Trans. R. Soc. Lond. A 360 (2002), 2245-2336

[8] G. Iooss, A. Mielke, Bifurcating time-periodic Solutions of Navier-Stokes Equations in Infinite Cylinders, J. Nonlinear Sci. 1 (1991), 1, 107-146

[9] K. Kirchgässner, Wave Solutions of Reversible Systems and Applications, J. Diff. Equ. 45 (1982), 113-127

[10] H. Ono, Algebraic solitary Waves in Stratified Fluids, J. Phys. Soc. Jpn, 39 (1975) 1082-1091

[11] C.A. Stuart, Bifurcation into Spectral Gaps, Bull. Belg. Math. Soc. Simon Stevin (1995), suppl., 59 pp.

[12] S.M. Sun, Existence of Solitary Internal Waves in a Two-Layer Fluid of Infinite Depth. Nonlinear Analysis 30 (1997) 5481-5490 